\title{
OBSERVATIONS OF THE ECONOMIC PRODUCTION QUANTITY MODEL WITH IMPERFECT QUALITY ITEMS WITH/WITHOUT INSPECTION
}

\author{
by \\ Yu Wei \\ Bachelor of Science in Mathematical Statistics, \\ East China Normal University, Shanghai, China, 1995 \\ Master of Management in Management of Science and Engineering, \\ Nanjing University, Nanjing, China, 2006
}

\author{
An MRP \\ presented to Ryerson University \\ in partial fulfillment of the \\ requirements for the degree of \\ Master of Engineering \\ in the Program of \\ Mechanical and Industrial Engineering
}

Toronto, Ontario, Canada, 2016

(C) Yu Wei 2016 


\section{Author's Declaration}

\section{AUTHOR'S DECLARATION FOR ELECTRONIC SUBMISSION OF A MRP}

I hereby declare that I am the sole author of this MRP. This is a true copy of the MRP, including any required final revisions.

I authorize Ryerson University to lend this MRP to other institutions or individuals for the purpose of scholarly research after 2019.

I further authorize Ryerson University to reproduce this MRP by photocopying or by other means, in total or in part, at the request of other institutions or individuals for the purpose of scholarly research.

I understand that my MRP may be made electronically available to the public. 


\section{Abstract}

Observations of the Economic Production Quantity Model with Imperfect Quality Items with/without Inspection

Author: Yu Wei

Master of Engineering in the Program of Mechanical and Industrial Engineering, Ryerson University, 2016

Three observations of the economic production quantity model with imperfect quality items with/without inspection are presented in this report.

Salameh and Jaber (2000) and Maddah and Jaber (2008) models do not meet their expected objective functions. The first observation tries to clarify the relationships among their expected objective functions and the actual ones through the derivation, the numerical simulation and statistical comparison. This report concludes s that both models are not very accurate, but capable.

Khan et al. (2011) and Khan et al. (2014) ignored the independent condition of properties of the expectation of random variables, which resulted in minor errors in both papers. The second and the third observations are the corrections of the models of two papers, respectively. Numerical comparisons show the differences between the original models and the revisions are minor, but significant. An example of a multi-factor sensitivity analysis is shown in this report. 


\section{Acknowledgement}

First of all, I appreciate my supervisor: Professor Mohamad Y. Jaber very much for his guidance, encouragement, knowledgeable advice, trust, and patience.

Secondly, I am grateful to Dr. Mehmood Khan for his guidance and kindness support.

Thirdly, I am thankful to all of my friends who helped me a lot.

Finally, thank my families: my husband, my son, my brother and my sisters for their love, patience, and unconditional support. 


\section{Table of Contents}

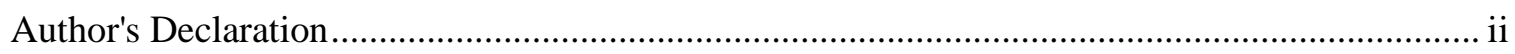

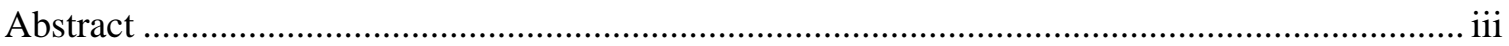

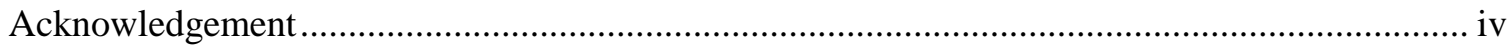

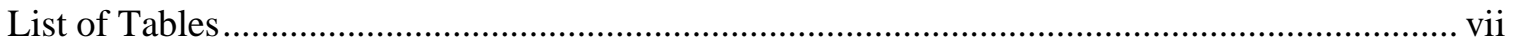

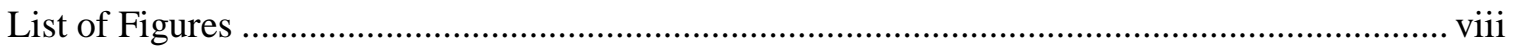

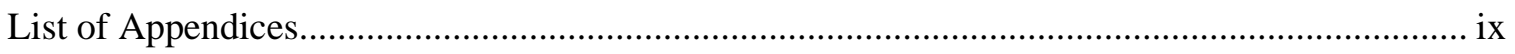

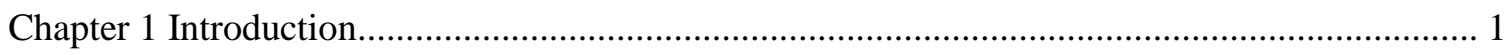

Chapter 2 Observation 1-- Comparison of the Models Between Salameh and Jaber (2000) and

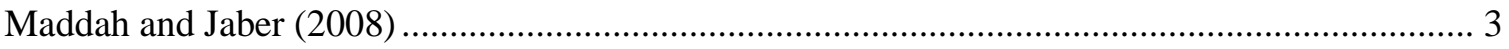

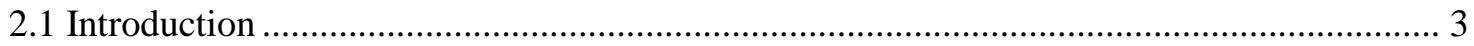

2.1.1 Review of Salameh and Jaber (2000) and Maddah and Jaber (2008) ............................ 3

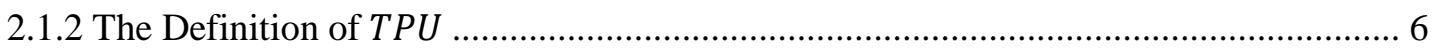

2.1.3 Remarks on the Models of Salameh and Jaber (2000) and Maddah and Jaber (2008)... 7

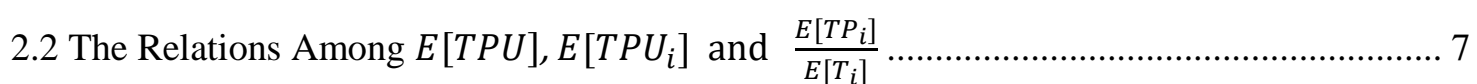

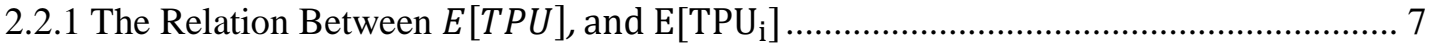

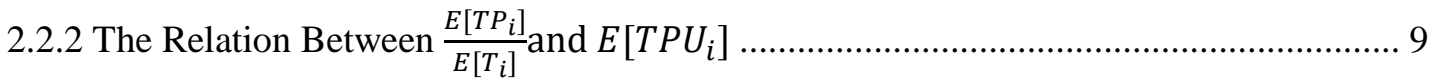

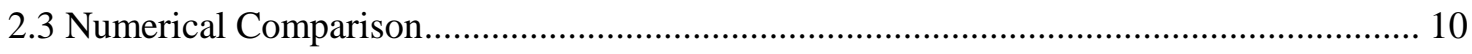

2.3.1 Comparison of $E\left[T P U\left(y_{S J}^{*}\right)\right]$ and $E\left[T P U_{i}\right]$ in Salameh and Jaber (2000).................... 10

2.3.2 Comparison of $E\left[T P U\left(y_{M J}^{*}\right)\right]$ and $\frac{E\left[T P_{i}\right]}{E\left[T_{i}\right]}$ in Maddah and Jaber (2008)....................... 12

2.3.3 Comparison of $E\left[T P U\left(y_{S J}^{*}\right)\right]$ of Salameh and Jaber (2000) and $E\left[T P U\left(y_{M J}^{*}\right)\right]$ of

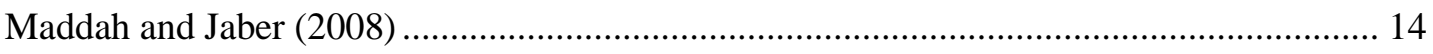

Chapter 3 Observation 2-- A Note on Khan et al. (2011)........................................................... 19

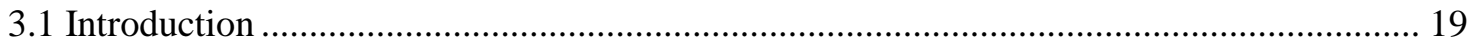

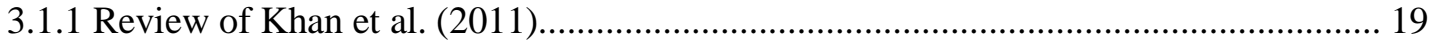

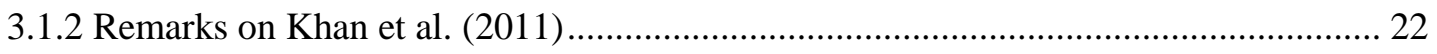

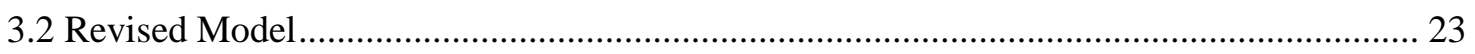

3.3 Numerical Comparison of the Model and Sensitivity Analysis ........................................ 26

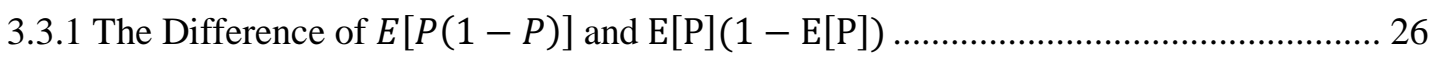

3.3.2 Numerical Comparison of Khan et al. (2011) to the Revised Model ........................... 27

3.3.3 Numerical Comparison of $\operatorname{TPU}_{\mathrm{Khan}(2011)}\left(\mathrm{y}_{\mathrm{Khan}(2011)}^{*}\right)$ and $\operatorname{TPU}_{\mathrm{Khan}(2011)}\left(\mathrm{y}_{\text {revised }}^{*}\right)$ 


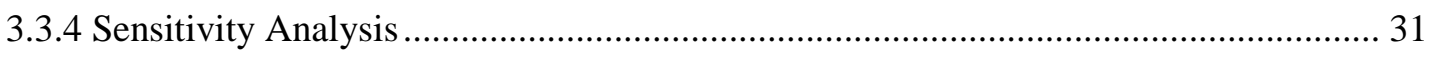

Chapter 4 Observation 3-- A Note on Khan et al. (2014)............................................................. 35

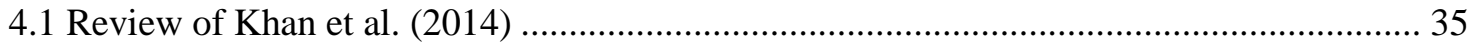

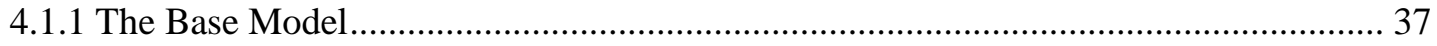

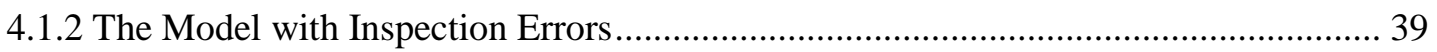

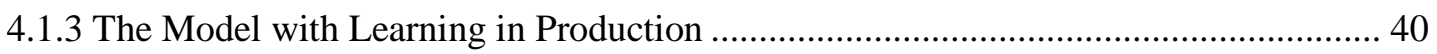

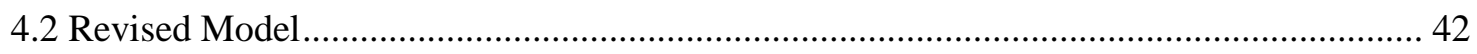

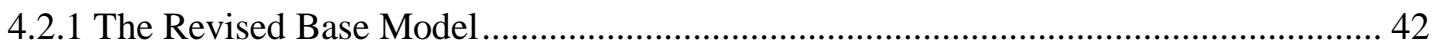

4.2.2 The Revised Model with Inspection Errors ....................................................................... 44

4.2.3 The Revised Model with Learning in Production........................................................... 47

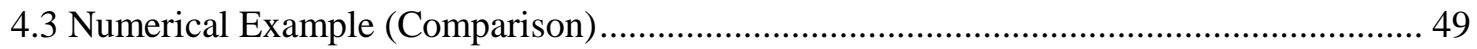

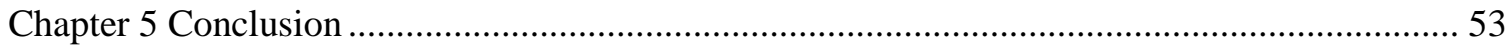

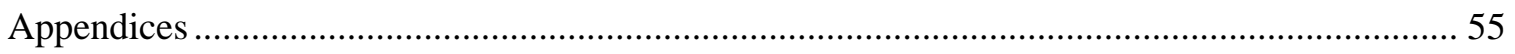

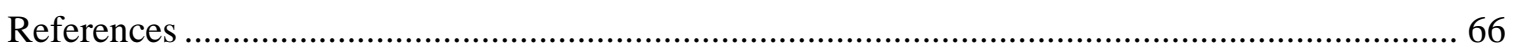




\section{List of Tables}

Table 1 Comparison of $E\left[T P U\left(y_{S J}^{*}\right)\right]$ and $E\left[T P U_{i}\right]$ in Salameh and Jaber (2000)

Table 2 Comparison of $E\left[T P U\left(y_{M J}^{*}\right)\right]$ and $\frac{E\left[T P_{i}\right]}{E\left[T_{i}\right]}$ in Maddah and Jaber (2008) 14

Table 3 Comparison of $E\left[T P U\left(y_{S J}^{*}\right)\right]$ of Salameh and Jaber (2000) and

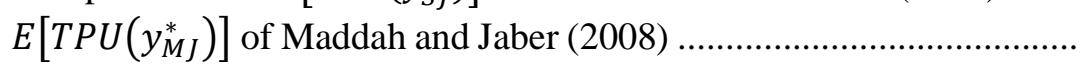

Table 4 Comparison of $y_{S J}^{*}$ of Salameh and Jaber (2000) and $y_{M J}^{*}$ of Maddah

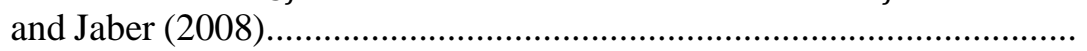

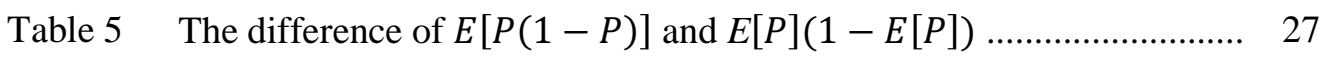

Table 6 Comparison of the optimal lot size $y^{*}$ and the expected total profit per unit time $E[T P U]$ between Khan et al. (2011) and revised model .

Table 7 Numerical Comparison of $T P U_{K h a n(2011)}\left(y_{K h a n(2011)}^{*}\right)$ and

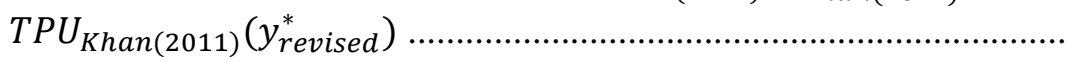

Table 8 The effects of only changing one factor at a time and the effect of changing three factors simultaneously....

Table 9 Numerical comparison of Khan et al. (2014) and revised model

$$
\left(E[\gamma]=0.02, E\left[m_{1}\right]=0.02 \text {, and } E\left[m_{2}\right]=0.02\right)
$$

Table 10 Numerical comparison of Khan et al. (2014) and revised model

$$
\left(E[\gamma]=0.2, E\left[m_{1}\right]=0.2 \text {, and } E\left[m_{2}\right]=0.2\right)
$$

Table 11 Numerical comparison of Khan et al. (2014) and revised model

$$
\left(E[\gamma]=0.5, E\left[m_{1}\right]=0.5 \text {, and } E\left[m_{2}\right]=0.5\right)
$$




\section{List of Figures}

Figure 1 Comparison of $E[T P U(y=1454)]$ and $E\left[T P U\left(y^{*}\right)\right]$ for

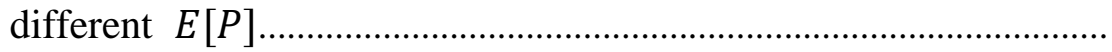

Table 2 Figure 2 The difference of expected annual profits $E[T P U(y=$ $1454)]$ and $E\left[T P U\left(y^{*}\right)\right]$ for different $E[P] \ldots \ldots \ldots \ldots \ldots \ldots \ldots \ldots \ldots$ 


\section{List of Appendices}

Appendix 1 Matlab code for the numerical comparison of the models of Salameh and Jaber (2000) and Maddah and Jaber (2008) ............ 55

Appendix 2 Matlab code for comparison of the optimal lot size and the expected total profit per unit time between Khan et al. (2011) and revised model.........................................................................

Appendix 3 Matlab code for Numerical Comparison of TPU $U_{K h a n(2011)}($

$\left.y_{\text {Khan(2011) }}^{*}\right)$ and TPU $U_{\text {Khan(2011) }}\left(y_{\text {revised }}^{*}\right)$

Appendix 4 Matlab code for the numerical comparison of Khan et al. (2014) and revised model.................................................................. 61

Appendix 4.1 Matlab code for the calculation of the model of Khan et al. (2014)

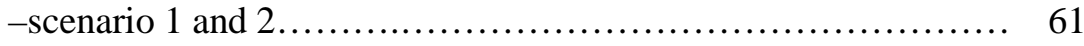

Appendix 4.2 Matlab code for the calculation of the model of Khan et al. (2014)

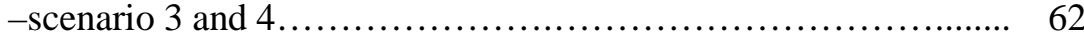

Appendix 4.3 Matlab code for the calculation of the revised model of Khan et al.

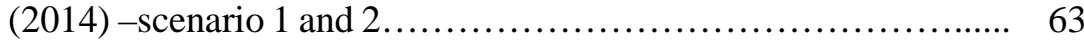

Appendix 4.4 Matlab code for the calculation of the revised model of Khan et al. (2014) -scenario 3 and 4 


\section{Chapter 1 Introduction}

The assumptions of the traditional EPQ/EOQ model are rigorous. Salameh and Jaber (2000) extend the traditional EPQ/EOQ model by taking imperfect quality items into account. Maddah and Jaber (2008) mention that there is a flaw in the method of evaluating the expected profit per unit of time, $E[T P U]$, in Salameh and Jaber (2000). Maddah and Jaber (2008) argue that the approach in modelling of the $E[T P U]$ in Salameh and Jaber (2000) is not accurate since they did not use the renewal reward theory in determining $E[T P U]$.

The work of Salameh and Jaber (2000) and Maddah and Jaber (2008) received considerable attention by several researchers, such as Vörös (2013), Khan et al. (2011), Khan et al. (2014), Zhou et al. (2016), and Rezaei (2016), to name some. This report shows that the two models are not the exact expression of $E[T P U]$, but rather approximate the expressions of $E[T P U]$. The definition of the total profit per unit of time, $T P U$, needs to be classified, and it is meaningful to discuss how the objective functions of the two models relate.

Khan et al. (2011) extend the EOQ model by considering items of imperfect quality and inspection errors. Khan et al. (2014) established a model for an integrated supply chain taking quality inspection and learning in production into consideration. Both studies apply the same method provided in Maddah and Jaber (2008). A wide attention has been given to both papers by Khan et al. (e.g., Chang et al. (2016), Azadeh et al. (2016), and Hou et al. (2015)).

Khan et al. (2011, 2014) neglect the independence of random variables, (for

example, the cycle length $T=\frac{y(1-P)\left(1-m_{1}\right)}{D}$ is relevant to the percentage of defective 
items $P$ ) when the properties of the expectation of random variables are applied, which results in minor errors. So, the two models need to be revised.

There are three observations in this report. In Chapter 2, a comparison of the models, Salameh and Jaber (2000) and Maddah and Jaber (2008), is presented. The definition of the total profit per unit time, $T P U$, the relationship among expected profit per unit time, $E[T P U]$,the real objective functions of the two models are discussed, and a numerical comparison is provided. In Chapter 3, the model of Khan et al. (2011) is reviewed and the error is highlighted. Revised model and numerical comparison are given in this chapter. Examples of a multi-factor sensitivity analysis are also provided. In Chapter 4, the model of Khan et al. (2014) is reviewed and corrected. The results of the revised model are compared with those of Khan et al. (2014). A conclusion is given in Chapter 5. 


\section{Chapter 2 Observation 1-- Comparison of the Models Between Salameh and Jaber (2000) and Maddah and Jaber (2008)}

\subsection{Introduction}

This section provides a brief review of the models of Salameh and Jaber (2000) and Maddah and Jaber (2008), then defines the total profit per unit time, $T P U$. This chapter concludes with a remark on the two models.

\subsubsection{Review of Salameh and Jaber (2000) and Maddah and Jaber (2008)}

The following notation are used in both Salameh and Jaber (2000) and Maddah and Jaber (2008).

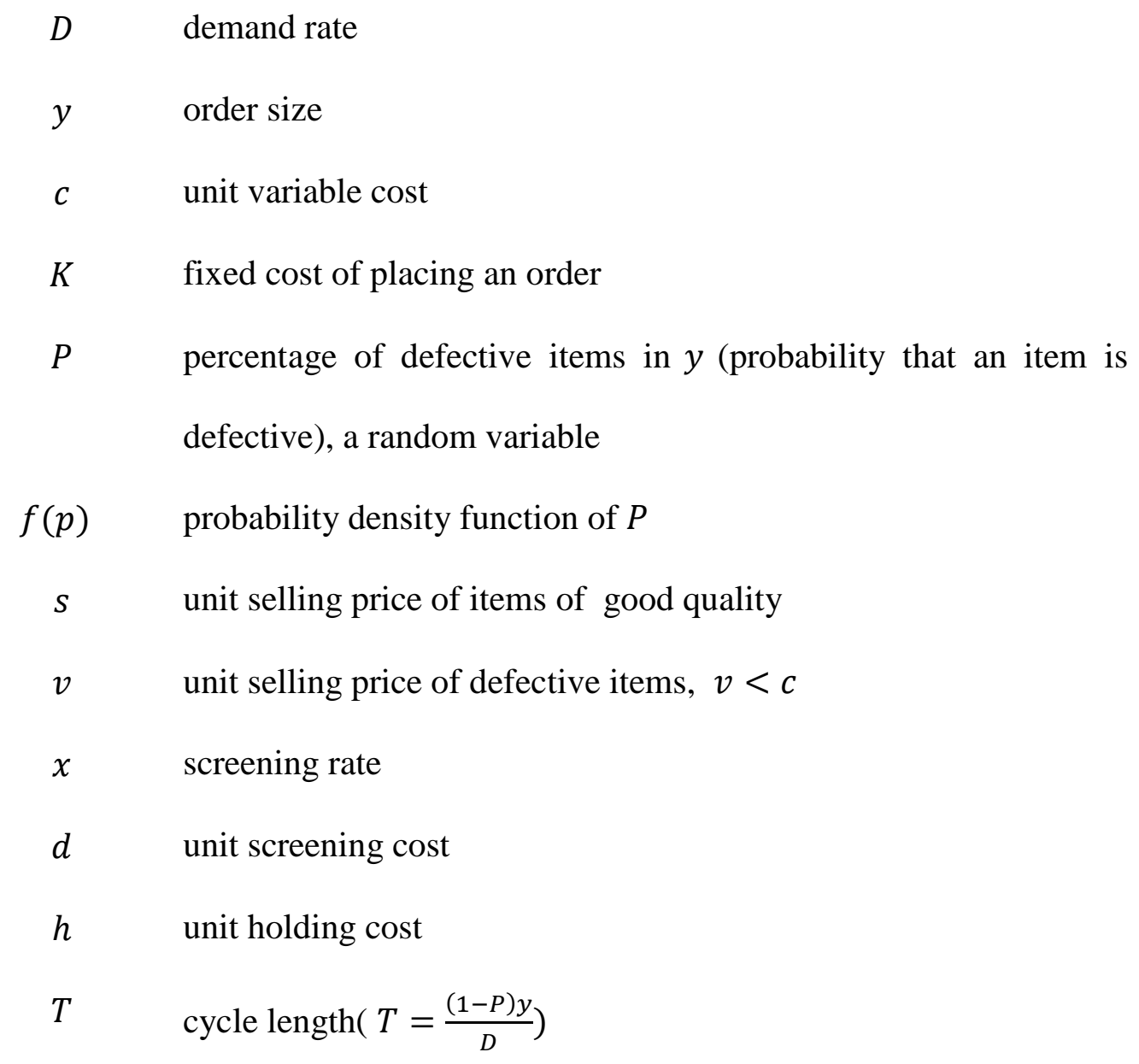


$M \quad$ expected value of $1 /(1-P)$

The following notation are also used.

$\begin{array}{cl}i & \text { index of cycle } \\ M(t) & \text { the number of renewals (cycles) for a finite time } t \\ T P_{i} & \text { total profit of ith cycle } \\ T P U_{i} & \text { total profit per unit time of ith cycle } \\ T P U(t) & \text { total profit per unit time in a finite time } t\end{array}$

Salameh and Jaber (2000) assume that: (1) defective items are sold as a single batch at a discounted price; (2) a 100\% percent screening process of the lot is conducted at a rate of $x$ units per unit of time; and (3) items of poor quality are kept in stock and sold prior to receiving the next shipment (by the end of the screening period) as a single batch at a discounted price of $v$ per unit. The profit per cycle is given by Salameh and Jaber (2000) as

$$
T P(y)=s y(1-P)+v y P-K-c y-d y-h\left(\frac{\left[y(1-P]^{2}\right.}{2 D}+\frac{P y^{2}}{x}\right)
$$

the cycle length is

$$
T=\frac{(1-P) y}{D}
$$

From Eq. (2.1) and Eq. (2.2), the profit per unit of time is given as

$$
T P U(y)=T P(y) / T
$$

Eq. (2.3) can be written as

$$
\begin{aligned}
\operatorname{TPU}(y)=D( & \left(s-v+h \frac{y}{x}\right)+D\left(v-\frac{h y}{x}-c-d-\frac{K}{y}\right) \times \frac{1}{1-P} \\
& -\frac{h y(1-P)}{2}
\end{aligned}
$$


So, the expected profit per unit of time is derived in Salameh and Jaber (2000) as

$$
E[T P U(y)]=E\left[\frac{T P(y)}{T}\right]
$$

, which becomes

$$
\begin{aligned}
E[T P U(y)]= & D\left(s-v+h \frac{y}{x}\right)+D\left(v-\frac{h y}{x}-c-d-\frac{K}{y}\right) \\
& \times E\left[\frac{1}{1-P}\right]-\frac{h y(1-E[P])}{2}
\end{aligned}
$$

The optimal order quality that maximizes Eq. (2.6) is given in Salameh and Jaber (2000) as

$$
y_{S J}^{*}=\sqrt{\frac{2 K D E[1 /(1-P)]}{h[1-E[P]-2 D(1-E[1 /(1-P)]) / x]}}
$$

Note: There is a typographical error in the expression of the optimal order quality of Salameh and Jaber (2000), which is corrected by Cardenas-Barron (2000). The "2" in the denominator is missing in Salameh and Jaber (2000). CardenasBarron (2000) conclud that this error does not affect the behavior of the model nor the results.

Maddah and Jaber (2008) mention that there is a flaw in Eq. (2.5) of Salameh and Jaber (2000), which is not accurate (see, for example, Wolff (1989), p. 60). The authors derive the expected profit per unit time by applying renewal-reward theorem as

$$
E[T P U(y)]=\frac{E[T P(y)]}{E[T]}
$$

Then, the optimal order quantity is obtained by differentiating the expression of $E[T P U(y)]$ which 


$$
y_{M J}^{*}=\sqrt{\frac{2 K D}{h\left[E\left[(1-P)^{2}\right]+2 E[P] D / x\right]}}
$$

\subsubsection{The Definition of $T P U$}

For a finite time $t, t=\sum_{i=1}^{M(t)} T_{i}$ only when the last cycle is completed. When the last cycle is not completed, $t \neq \sum_{i=1}^{M(t)} T_{i}$. So

$T P U(t)=\frac{T P(t)}{t} \neq \frac{T P(t)}{T}$

Then Eq. (2.5) is not accurate, i.e.

$E[T P U(t)] \neq E\left[\frac{T P(t)}{T}\right]$

when the last cycle is not completed.

The definition of $T P U$ is given below:

1) If the reward is collected at the end of each cycle, the total profit per unit time in a finite time $t, T P U(t)$ is given as

$$
T P U(t)=\frac{T P(t)}{t}=\frac{1}{t} \sum_{i=1}^{M(t)} T P_{i}=\frac{1}{t} \sum_{i=1}^{M(t)} T P U_{i} \cdot T_{i}
$$

2) If the reward is collected at the beginning of each cycle, then

$T P U(t)=\frac{T P(t)}{t}=\frac{1}{t} \sum_{i=1}^{M(t)+1} T P_{i}=\frac{1}{t} \sum_{i=1}^{M(t)+1} T P U_{i} \cdot T_{i}$

3) If the reward is uniformly collected over a cycle,

$$
T P U(t)=\frac{T P(t)}{t}=\frac{1}{t}\left\{\sum_{i=1}^{M(t)} T P_{i}+T P_{M(t)+1} \cdot \frac{\sum_{i=1}^{M(t)+1} T_{i}-t}{T_{M(t)+1}}\right\}
$$




\subsubsection{Remark on the Models of Salameh and Jaber (2000) and Maddah and Jaber (2008)}

The renewal-reward theorem is a limit theorem instead of an accurate one (please refer to in Ross (1996), P.133 or Wolff (1988), P. 59). Because of this fact, and the models of Salameh and Jaber (2000) and Maddah and Jaber (2008) only consider one order cycle instead of all cycles, the models of both papers become approximations of the true function. So, to be more accurate, the objective function of the model of Salameh and Jaber (2000) should be written as $E\left[T P U_{i}\right]=$ $E\left[T P_{i} / T_{i}\right]$ instead of $E[T P U]$, where $i$ is the index of ith cycle. Similarly, the objective function of Maddah and Jaber (2008) should be written as $E\left[T P_{i}\right] / E\left[T_{i}\right]$. So, the relations among $E[T P U], E\left[T P U_{i}\right]$ and $\frac{E\left[T P_{i}\right]}{E\left[T_{i}\right]}$ are discussed next.

2.2 The Relations Among $E[T P U], E\left[T P U_{i}\right]$ and $\frac{E\left[T P_{i}\right]}{E\left[T_{i}\right]}$

\subsubsection{The Relation Between $E[T P U]$, and $E\left[T P U_{i}\right]$}

Because

$T P U_{i}=\frac{T P_{i}}{T_{i}}$

$T P_{i}=T P U_{i} * T_{i}$

$T P U_{i}$ and $T_{i}$ are i.i.d. with expectations of $E\left[T P U_{i}\right]$ and $E\left[T_{i}\right]$, respectively. Let

$$
\begin{aligned}
& T P U_{i}=E\left[T P U_{i}\right]+\varepsilon_{i} \\
& T_{i}=E\left[T_{i}\right]+\eta_{i}
\end{aligned}
$$

where $\varepsilon_{i}$ and $\eta_{i}$ are i.i.d. respectively, and $E\left[\varepsilon_{i}\right]=0, E\left[\eta_{i}\right]=0$ for $i=$ $1, \ldots, M(t)$. Without loss of generality, assuming the reward is collected at the end and $M(t) \geq 2$ 


$$
\begin{aligned}
T P U(t)=\frac{T P(t)}{t} & =\frac{1}{t} \sum_{i=1}^{M(t)} T P_{i}=\frac{1}{t} \sum_{i=1}^{M(t)} T P U_{i} * T_{i} \\
& =\frac{1}{t} \sum_{i=1}^{M(t)}\left[\left(E\left[T P U_{i}\right]+\varepsilon_{i}\right)\left(E\left[T_{i}\right]+\eta_{i}\right)\right]
\end{aligned}
$$

Because $T P U_{i}$ is i.i.d., $E\left[T P U_{i}\right]$ are the same for different $i$, to simplify the expression, let $E\left[T P U_{i}\right]=a$. Similarly, let $E\left[T_{i}\right]=b$

$$
\begin{aligned}
T P U(t) & =\frac{1}{t} \sum_{i=1}^{M(t)}\left[\left(a+\varepsilon_{i}\right)\left(b+\eta_{i}\right)\right] \\
& =\frac{1}{t} \sum_{i=1}^{M(t)}\left[\left(a+\varepsilon_{i}\right)\left(b+\eta_{i}\right)\right]=\frac{1}{t} \sum_{i=1}^{M(t)}\left[a b+a \eta_{i}+b \varepsilon_{i}+\varepsilon_{i} \eta_{i}\right] \\
& =\frac{1}{t}\left(a b * M(t)+a \sum_{i=1}^{M(t)} \eta_{i}+b \sum_{i=1}^{M(t)} \eta_{i}+\sum_{i=1}^{M(t)} \varepsilon_{i} \eta_{i}\right)
\end{aligned}
$$

Because $\lim _{\mathrm{t} \rightarrow \infty} \frac{E[M(t)]}{t}=\frac{1}{E\left[T_{i}\right]}=\frac{1}{b}$ (the Elementary Renewal Theorem (Wolff (1988), page 59) and Wald’s equation (Wolff (1988), page 98)

$$
\begin{aligned}
\lim _{\mathrm{t} \rightarrow \infty} E[T P U(t)] & =\frac{1}{t}\left(a b * \frac{t}{b}+0+0+\frac{t}{b} E\left[\varepsilon_{i} \eta_{i}\right]\right)=a+\frac{1}{b} E\left[\varepsilon_{i} \eta_{i}\right] \\
= & E\left[T P U_{i}\right]+\frac{E\left[\varepsilon_{i} \eta_{i}\right]}{E\left[T_{i}\right]}
\end{aligned}
$$

Because Eq. (2.13) and Eq. (2.14), then

$$
\begin{aligned}
\varepsilon_{i} & =T P U_{i}-E\left[T P U_{i}\right] \\
\eta_{i} & =T_{i}-E\left[T_{i}\right]
\end{aligned}
$$

, respectively.

By substituting Eq. (2.16) and Eq. (2.17), we get 


$$
\begin{aligned}
\lim _{t \rightarrow \infty} E[T P U(t)] & =E\left[T P U_{i}\right]+\frac{E\left[\left(T P U_{i}-E\left[T P U_{i}\right]\right)\left(T_{i}-E\left[T_{i}\right]\right)\right]}{E\left[T_{i}\right]} \\
= & E\left[T P U_{i}\right]+\frac{\operatorname{COV}\left(T P U_{i}, T_{i}\right)}{E\left[T_{i}\right]}
\end{aligned}
$$

2.2.2 The Relation Between $\frac{E\left[T P_{i}\right]}{E\left[T_{i}\right]}$ and $E\left[T P U_{i}\right]$

$$
\begin{aligned}
E\left[T P_{i}\right] & \left.=E\left[\left(E\left[T P U_{i}\right]+\varepsilon_{i}\right)\left(E\left[T_{i}\right]+\eta_{i}\right)\right]\right] \\
& =E\left[E\left[T P U_{i}\right] * E\left[T_{i}\right]\right]+E\left[E\left[T P U_{i}\right] * \eta_{i}\right]+E\left[E\left[T_{i}\right] * \varepsilon_{i}\right]+E\left[\varepsilon_{i} \eta_{i}\right] \\
& =E\left[T P U_{i}\right] * E\left[T_{i}\right]+E\left[\varepsilon_{i} \eta_{i}\right]
\end{aligned}
$$

So,

$$
\frac{E\left[T P_{i}\right]}{E\left[T_{i}\right]}=E\left[T P U_{i}\right]+\frac{E\left[\varepsilon_{i} \eta_{i}\right]}{E\left[T_{i}\right]}=E\left[T P U_{i}\right]+\frac{\operatorname{COV}\left(T P U_{i}, T_{i}\right)}{E\left[T_{i}\right]}
$$

According to the renewal-reward theorem

$$
\lim _{t \rightarrow \infty} E[T P U(t)]=\frac{E\left[T P_{i}\right]}{E\left[T_{i}\right]}
$$

In conclusion, the relation between $E[T P U]$, and $E\left[T P U_{i}\right]$, and subsequently $\frac{E\left[T P_{i}\right]}{E\left[T_{i}\right]}$ is given as

$$
\lim _{t \rightarrow \infty} E[T P U(t)]=\frac{E\left[T P_{i}\right]}{E\left[T_{i}\right]}=\mathrm{E}\left[T P U_{i}\right]+\frac{\operatorname{COV}\left(T P U_{i}, T_{i}\right)}{E\left[T_{i}\right]}
$$

So, when $\operatorname{COV}\left(T P U_{i}, T_{i}\right)$ is equal to $0, \lim _{\mathrm{t} \rightarrow \infty} E[T P U(t)]=\frac{E\left[T P_{i}\right]}{E\left[T_{i}\right]}=$ $E\left[T P U_{i}\right]$. A special case occurs, when $T_{1}=T_{2}=\cdots T_{M(t)}=E\left[T_{i}\right]=T$, $E\left[T_{i}-E\left[T_{i}\right]\right]=0$, i.e. $T$ is a constant instead of being a random variable, where $\lim _{\mathrm{t} \rightarrow \infty} E[T P U(t)]$ is also equal to $E\left[T P U_{i}\right]$. But, if $\operatorname{COV}\left(T P U_{i}, T_{i}\right)$ is not equal to 0 , then $\lim _{\mathrm{t} \rightarrow \infty} E[T P U(t)]=\frac{E\left[T P_{i}\right]}{E\left[T_{i}\right]} \neq E\left[T P U_{i}\right]$. The difference between $\lim _{\mathrm{t} \rightarrow \infty} E[T P U(t)]$ 
and $E\left[T P U_{i}\right]$ is $\frac{\operatorname{COV}\left(T P U_{i}, T_{i}\right)}{E\left[T_{i}\right]}$, which is not considered in Salameh and Jaber (2000).

It is worth mentioning that $\frac{E\left[T P_{i}\right]}{E\left[T_{i}\right]}$ is only equal to the limit of $E[T P U]$ instead of $E[T P U]$ itself.

\subsection{Numerical Comparison}

In this section, three numerical comparisons are provided. The first compares $E\left[T P U\left(y_{S J}^{*}\right)\right]$ and $E\left[T P U_{i}\right]$ in Salameh and Jaber (2000); the second compares $E\left[T P U\left(y_{M J}^{*}\right)\right]$ and $\frac{E\left[T P_{i}\right]}{E\left[T_{i}\right]}$ in Maddah and Jaber (2008); and the third compares $E\left[T P U\left(y_{S J}^{*}\right)\right]$ of Salameh and Jaber (2000) and $E\left[T P U\left(y_{M J}^{*}\right)\right]$ of Maddah and Jaber (2008), respectively.

\subsubsection{Comparison of $E\left[T P U\left(y_{S J}^{*}\right)\right]$ and $E\left[T P U_{i}\right]$ in Salameh and Jaber (2000)}

The expected objective function of Salameh and Jaber (2000)'s model is $E\left[T P U\left(y_{S J}^{*}\right)\right]$. And the actual objective function of Salameh and Jaber (2000)'s model is $E\left[T P U_{i}\right]$. To compare them, the following hypothesis is tested by using the same parameter values from Salameh and Jaber (2000) and Maddah and Jaber (2008).

$H_{01}: E\left[T P U\left(y_{S J}^{*}\right)\right]=E\left[T P U_{i}\right]$

$H_{a 1}: E\left[T P U\left(y_{S J}^{*}\right)\right] \neq E\left[T P U_{i}\right]$

The parameters and their values are: $D=50,000$ units/year, $c=\$ 25 /$ unit, $K$ $=\$ 100 /$ cycle,$\quad s=\$ 50 /$ unit,$\quad d=\$ 0.5 /$ unit,$\quad x=1$ unit $/ \mathrm{min}=175,200$ units $/$ year,$\quad h$ $=\$ 5 /$ unit/year, $v=\$ 20 /$ unit. The fraction of imperfect quality items, $P$, is uniformly 
distributed on $(a, b), 0<a<b<1$, i.e., $P \sim U(a, b)$ and $a=0$.

A Matlab code (please see Appendix 1) is programed to simulate the comparison with the steps given below:

Step 1: Calculate the optimal order size $y_{S J}^{*}$ by using Eq. (2.7).

Step 2: Calculate the expected total profit per unit time of $i$ th cycle $E\left[T P U_{i}\right]$ by using Eq. (2.6).

Step 3: Randomly generate the fraction of imperfect quality items $P \sim U(a, b)$. Calculate the annual total profit, i.e. total profit per unit of time (one year) $T P U\left(y_{S J}^{*}\right)$ by using Eq. (2.1)and Eq.(2.10).

Step 4: Repeat step 3 for $n=1000$ times.

Step 5: Test $H_{01}: E\left[T P U\left(y_{S J}^{*}\right)\right]=E\left[T P U_{i}\right]$ by using t-test.

In step 5, one sample t-test is chosen because it is a one mean test for a large sample size (Thank to the central limit theorem (CLM)) with an unknown variance. The approach of the t-test is introduced below:

1) If a random sample of size $n: X_{1}, X_{2}, \cdots, X_{n}$

2) $H_{0}: E[X]=\mu=\mu_{0}$

$$
H_{a}: E[X]=\mu \neq \mu_{0}
$$

3) Level of significance is $\alpha$,

4) Test statistic is

$t=\frac{\bar{X}-\mu_{0}}{S / \sqrt{n}}$

where

$$
S=\sqrt{\frac{\sum_{i=1}^{n}\left(X_{i}-\bar{X}\right)^{2}}{n-1}}
$$


5) Rejection region is $=\{t<-t \alpha / 2\} \cup\{t>t \alpha / 2\}$, where $t \alpha / 2$ is the percentile of t-student random variable. The degree of freedom used is $n-1$. It can also be judged by the P-value of the output of software. The P-value is less than $\alpha$, which means that the null hypothesis can be rejected at $\alpha$ level.

When $P \sim U(a, b)$, then $E[P]=\frac{b-a}{2}$. In reality, the percentage of defective items $P$ is very small, so $b$ is chosen to be less than or equal to 0.50 (although this number is very high). For different $b$, the comparison is shown in Table 1 , where $\overline{T P U}\left(y_{S J}^{*}\right)$ is an estimator of $E\left[T P U\left(y_{S J}^{*}\right)\right]$ calculated by using the model of Salameh and Jaber (2000) and

$$
\begin{aligned}
& \Delta_{1}=E\left[T P U_{i}\right]-\overline{T P U}\left(y_{S J}^{*}\right) \\
& \Delta_{1}(\%)=\frac{E\left[T P U_{i}\right]-\overline{T P U}\left(y_{S J}^{*}\right)}{\overline{T P U}\left(y_{S J}^{*}\right)} \times 100 \%
\end{aligned}
$$

Table 1 Comparison of $E\left[T P U\left(y_{S J}^{*}\right)\right]$ and $E\left[T P U_{i}\right]$ in Salameh and Jaber (2000)

\begin{tabular}{c|c|r|r|c|r|r|l}
\hline$b$ & $E[P]$ & $\overline{T P U}\left(y_{S J}^{*}\right)$ & $E\left[T P U_{i}\right]$ & $\begin{array}{c}95 \% \text { CI of } \\
T P U\left(y_{S J}^{*}\right)\end{array}$ & $\Delta_{1}$ & $\Delta_{1}(\%)$ & P-value \\
\hline 0.01 & 0.005 & 1206074 & 1216534 & $(1206030,1206119)$ & 10460 & 0.87 & 0 \\
\hline 0.04 & 0.020 & 1196611 & 1212235 & $(1196432,1196791)$ & 15624 & 1.31 & 0 \\
\hline 0.07 & 0.035 & 1187067 & 1207752 & $(1186711,1187424)$ & 20685 & 1.74 & 0 \\
\hline 0.10 & 0.050 & 1186785 & 1203071 & $(1186102,1187467)$ & 16287 & 1.37 & $2.15 \mathrm{E}-254$ \\
\hline 0.15 & 0.075 & 1184595 & 1194790 & $(1183987,1185202)$ & 10195 & 0.86 & $1.13 \mathrm{E}-161$ \\
\hline 0.20 & 0.100 & 1174394 & 1185837 & $(1173750,1175039)$ & 11442 & 0.97 & $1.41 \mathrm{E}-174$ \\
\hline 0.25 & 0.125 & 1166081 & 1176114 & $(1165439,1166724)$ & 10032 & 0.86 & $5.86 \mathrm{E}-146$ \\
\hline 0.30 & 0.150 & 1156270 & 1165502 & $(1155586,1156954)$ & 9232 & 0.80 & $1.39 \mathrm{E}-117$ \\
\hline 0.40 & 0.200 & 1136121 & 1140986 & $(1135388,1136853)$ & 4865 & 0.43 & $5.35 \mathrm{E}-36$ \\
\hline 0.50 & 0.250 & 1112792 & 1110567 & $(1111855,1113729)$ & -2224 & -0.20 & $3.62 \mathrm{E}-06$ \\
\hline
\end{tabular}

It can be seen from Table 1 that the differences between $\overline{T P U}\left(y_{S J}^{*}\right)$ and 
$E\left[T P U_{i}\right]$ are very small. But all differences are significant, which means the differences are systematic.

\subsubsection{Comparison of $E\left[T P U\left(y_{M J}^{*}\right)\right]$ and $\frac{E\left[T P_{i}\right]}{E\left[T_{i}\right]}$ in Maddah and Jaber (2008)}

Similar to Section 2.3.1, the expected objective function of Maddah and Jaber (2008)'s model is $E\left[T P U\left(y_{M J}^{*}\right)\right]$, and the actual objective function in Maddah and Jaber (2008) is $\frac{E\left[T P_{i}\right]}{E\left[T_{i}\right]}$.

To compare the difference of them, the following hypothesis is tested by using the same parameters from Maddah and Jaber (2008).

$$
\begin{aligned}
& H_{02}: E\left[T P U\left(y_{M J}^{*}\right)\right]=\frac{E\left[T P_{i}\right]}{E\left[T_{i}\right]} \\
& H_{a 2}: E\left[T P U\left(y_{M J}^{*}\right)\right] \neq \frac{E\left[T P_{i}\right]}{E\left[T_{i}\right]}
\end{aligned}
$$

Matlab program is also in Appendix 1 and the steps are given below:

Step 1: Calculate the optimal order size $y_{M J}^{*}$ by using Eq. (2.9).

Step 2: Calculate $\frac{E\left[T P_{i}\right]}{E\left[T_{i}\right]}$ by using Eq. (2.8).

Step 3: Randomly generate the fraction of imperfect quality items. $P \sim U(a, b)$. Calculate the annual total profit i.e. total profit per unit time (one year) $T P U\left(y_{M J}^{*}\right)$ by using Eq. (2.1)and Eq.(2.10).

Step 4: Repeat step 3 for $n=1000$ times.

Step 5: Test $H_{02}: E\left[T P U\left(y_{M J}^{*}\right)\right]=\frac{E\left[T P_{i}\right]}{E\left[T_{i}\right]}$ by using t-test.

The results are shown in Table 2. 
Table 2 Comparison of $E\left[T P U\left(y_{M J}^{*}\right)\right]$ and $\frac{E\left[T P_{i}\right]}{E\left[T_{i}\right]}$ in Maddah and Jaber (2008)

\begin{tabular}{r|r|r|r|r|r|r|r}
\hline$b$ & $E[P]$ & $\overline{T P U}\left(y_{M J}^{*}\right)$ & $\frac{E\left[T P_{i}\right]}{E\left[T_{i}\right]}$ & $\begin{array}{c}95 \% \mathrm{CI} \text { of } \\
T P U\left(y_{M J}^{*}\right)\end{array}$ & $\Delta_{2}$ & $\Delta_{2}(\%)$ & P-value \\
\hline 0.01 & 0.005 & 1207460 & 1216537 & $(1207415,1207505)$ & 9077 & 0.75 & 0 \\
\hline 0.04 & 0.020 & 1202172 & 1212274 & $(1201992,1202352)$ & 10102 & 0.84 & 0 \\
\hline 0.07 & 0.035 & 1196146 & 1207878 & $(1195815,1196476)$ & 11732 & 0.98 & 0 \\
\hline 0.10 & 0.050 & 1190449 & 1203341 & $(1189988,1190910)$ & 12892 & 1.08 & $1.04 \mathrm{E}-303$ \\
\hline 0.15 & 0.075 & 1180633 & 1195448 & $(1180033,1181232)$ & 14815 & 1.25 & $8.00 \mathrm{E}-265$ \\
\hline 0.20 & 0.100 & 1173571 & 1187112 & $(1172928,1174214)$ & 13541 & 1.15 & $1.86 \mathrm{E}-218$ \\
\hline 0.25 & 0.125 & 1165548 & 1178294 & $(1164889,1166207)$ & 12745 & 1.09 & $6.77 \mathrm{E}-196$ \\
\hline 0.30 & 0.150 & 1156580 & 1168950 & $(1155907,1157253)$ & 12371 & 1.07 & $4.04 \mathrm{E}-183$ \\
\hline 0.40 & 0.200 & 1135668 & 1148491 & $(1134876,1136461)$ & 12823 & 1.13 & $1.57 \mathrm{E}-153$ \\
\hline 0.50 & 0.250 & 1112660 & 1125271 & $(1111675,1113645)$ & 12611 & 1.13 & $2.46 \mathrm{E}-108$ \\
\hline
\end{tabular}

where $\overline{T P U}\left(y_{M J}^{*}\right)$ is an estimator of $E\left[T P U\left(y_{M J}^{*}\right)\right]$ calculated by using the model of Maddah and Jaber (2008) and

$$
\begin{aligned}
& \Delta_{2}=\frac{E\left[T P_{i}\right]}{E\left[T_{i}\right]}-\overline{T P U}\left(y_{M J}^{*}\right) \\
& \Delta_{2}(\%)=\frac{\frac{E\left[T P_{i}\right]}{E\left[T_{i}\right]}-\overline{T P U}\left(y_{M J}^{*}\right)}{\overline{T P U}\left(y_{M J}^{*}\right)} * 100 \%
\end{aligned}
$$

It can be seen from Table 2 that the differences between $\overline{T P U}\left(y_{M J}^{*}\right)$ and $\frac{E\left[T P_{i}\right]}{E\left[T_{i}\right]}$ are very small too. And all differences are significant which means the differences are systematic.

\subsubsection{Comparison of $E\left[T P U\left(y_{S J}^{*}\right)\right]$ of Salameh and Jaber (2000) and $E\left[T P U\left(y_{M J}^{*}\right)\right]$ of Maddah and Jaber (2008)}

The expected objective function in Salameh and Jaber (2000) is 
$E\left[T P U\left(y_{S J}^{*}\right)\right]$, and the expected objective function of Maddah and Jaber (2008)'s model is $E\left[T P U\left(y_{M J}^{*}\right)\right]$. To compare $E\left[T P U\left(y_{S J}^{*}\right)\right]$ of Salameh and Jaber (2000) and $E\left[T P U\left(y_{M J}^{*}\right)\right]$ of Maddah and Jaber (2008), the following hypothesis is tested by using the same parameters from Salameh and Jaber (2000) and Maddah and Jaber (2008).

$H_{03}: E\left[T P U\left(y_{S J}^{*}\right)\right]=E\left[T P U\left(y_{M J}^{*}\right)\right]$

$H_{a 3}: E\left[T P U\left(y_{S J}^{*}\right)\right] \neq E\left[T P U\left(y_{M J}^{*}\right)\right]$

Matlab program is also in Appendix 1 and the steps are given below:

Step 1: Calculate the optimal order size $y_{S J}^{*}$ by using Eq. (2.7).

Step 2: Calculate the optimal order size $y_{M J}^{*}$ by using Eq. (2.9).

Step 3: Generate the random number of the fraction of imperfect quality items $P \sim U(a, b)$. Calculate the annual total profit i.e. total profit per unit time (one year) $T P U\left(y_{S J}^{*}\right)$ by using Eq. (2.1)and Eq.(2.10).

Step 4: Use the same random number of $P$ generated in step 3 and calculate the annual total profit i.e. total profit per unit time (one year) $T P U\left(y_{M J}^{*}\right)$ by using Eq. (2.1)and Eq.(2.10).

Step 5: Repeat step 3 and step 4for $n=1000$ times.

Step 6: Test $H_{03}: E\left[T P U\left(y_{S J}^{*}\right)\right]=E\left[T P U\left(y_{M J}^{*}\right)\right]$ by using paired sample ttest.

In step 6, paired sample t-test is chosen because there are two samples that have been matched or "paired". The approach is introduced below:

1) If two matched random samples of size $n: X_{1}, X_{2}, \cdots, X_{n}$ and $Y_{1}, Y_{2}, \cdots, Y_{n}$ 2) $H_{0}: E\left[X_{D}\right]=E[X-Y]=\mu_{0}$ 


$$
H_{a}: E\left[X_{D}\right]=E[X-Y] \neq \mu_{0}
$$

3) Level of significance is $\alpha$,

4) Test statistic is

$t=\frac{\bar{X}_{D}-\mu_{0}}{\frac{S_{D}}{\sqrt{n}}}$

where the average $\bar{X}_{D}$ and the standard deviation $S_{D}$ of those differences are used in the equation.

5) Rejection region is $=\{t<-t \alpha / 2\} \cup\{t>t \alpha / 2\}$, where $t \alpha / 2$ is the percentile of t-student random variable. The degree of freedom used is $n-1$, where $n$ represents the number of pairs. It can also be judged by the P-value of the output of software. The P-value is less than $\alpha$, which means that the null hypothesis can be rejected at $\alpha$ level.

Table 3 shows typical results of the first definition of TPU (the reward is collected at the end of each cycle) and the difference of optimal lot size of the two models is shown in Table 4.

where

$$
\Delta_{3}=\overline{T P U}\left(y_{M J}^{*}\right)-\overline{T P U}\left(y_{S J}^{*}\right)
$$

$$
\Delta_{3}(\%)=\frac{\overline{T P U}\left(y_{M J}^{*}\right)-\overline{T P U}\left(y_{S J}^{*}\right)}{\overline{T P U}\left(y_{S J}^{*}\right)} \times 100 \%
$$

And

$$
\begin{aligned}
& \Delta_{4}=y_{M J}^{*}-y_{S J}^{*} \\
& \Delta_{4}(\%)=\frac{y_{M J}^{*}-y_{S J}^{*}}{y_{S J}^{*}} \times 100 \%
\end{aligned}
$$


It can be seen from Table 4 that the differences in the values of $y_{S J}^{*}$ and $y_{M J}^{*}$, i.e. the optimal lot sizes for Salameh and Jaber (2000) and Maddah and Jaber (2008), increase when $b$ increases, which accords with the Proposition 8 of Vörös (2013). It is interesting to note in Table 3 that the differences between $\overline{T P U}\left(y_{S J}^{*}\right)$ and $\overline{T P U}\left(y_{M J}^{*}\right)$ are very small; $\left(\max \left\{\left|\Delta_{3}(\%)\right|\right\}=0.78 \%\right) . E\left[T P U\left(y_{M J}^{*}\right)\right]$ is significantly more than $E\left[T P U\left(y_{S J}^{*}\right)\right]$ when $b$ is small. $E\left[T P U\left(y_{M J}^{*}\right)\right]$ is more likely to be less than $E\left[\operatorname{TPU}\left(y_{S J}^{*}\right)\right]$ when $b$ is lager, where the values of the significance become unstable.

In conclusion, the two models are not very accurate, but capable. When the volatility of the percentage of defective items $P$ is small, which is the case in reality, the model of Maddah and Jaber (2008) is more accurate than that of Salameh and Jaber (2000). 
Table 3 Comparison of $E\left[T P U\left(y_{S J}^{*}\right)\right]$ of Salameh and Jaber (2000) and $E\left[T P U\left(y_{M J}^{*}\right)\right]$ of Maddah and Jaber (2008)

\begin{tabular}{r|r|r|r|r|r|r|r}
\hline \multicolumn{1}{c|}{$b$} & $E[P]$ & $T P U\left(y_{S J}^{*}\right)$ & $\overline{T P U}\left(y_{M J}^{*}\right)$ & \multicolumn{1}{c|}{$\Delta_{3}$} & $\Delta_{3}(\%)$ & \multicolumn{1}{l}{ P_value } & t_statistic \\
\hline 0.01 & 0.005 & 1206067 & 1207453 & 1386 & 0.11 & 0 & $5.40 \mathrm{E}+04$ \\
\hline 0.04 & 0.020 & 1196415 & 1201975 & 5560 & 0.46 & 0 & $1.32 \mathrm{E}+04$ \\
\hline 0.07 & 0.035 & 1187101 & 1196392 & 9291 & 0.78 & 0 & 74.58022544 \\
\hline 0.10 & 0.050 & 1186713 & 1190150 & 3437 & 0.29 & $5.75 \mathrm{E}-12$ & 6.970012336 \\
\hline 0.15 & 0.075 & 1184576 & 1180541 & -4035 & -0.34 & $4.63 \mathrm{E}-18$ & -8.829574034 \\
\hline $0.20(1)$ & 0.100 & 1174309 & 1172970 & -1339 & -0.11 & $1.75 \mathrm{E}-05$ & -4.315513023 \\
$0.20(2)$ & & 1173981 & 1173624 & -357 & -0.03 & 0.3023346 & -1.03197274 \\
$0.20(3)$ & & 1173638 & 1173712 & 74 & 0.01 & 0.8352869 & 0.207980007 \\
\hline $0.25(1)$ & 0.125 & 1166014 & 1165029 & -986 & -0.08 & 0.0046124 & -2.839337166 \\
$0.25(2)$ & & 1165031 & 1165220 & 189 & 0.02 & 0.5455244 & 0.604686704 \\
$0.25(3)$ & & 1165176 & 1165037 & -139 & -0.01 & 0.6774509 & -0.416066103 \\
\hline $0.30(1)$ & 0.150 & 1156819 & 1156637 & -182 & -0.02 & 0.7052240 & -0.378386256 \\
$0.30(2)$ & & 1157030 & 1155880 & -1151 & -0.10 & 0.0183785 & -2.361778159 \\
\hline $0.40(1)$ & 0.200 & 1135451 & 1135129 & -322 & -0.03 & 0.4046786 & -0.83364792 \\
$0.40(2)$ & & 1136654 & 1135265 & -1388 & -0.12 & $3.21 \mathrm{E}-04$ & -3.610033883 \\
\hline $0.50(1)$ & 0.250 & 1113329 & 1112965 & -363 & -0.03 & 0.4265102 & -0.795502409 \\
$0.50(2)$ & & 1113023 & 1111772 & -1250 & -0.11 & 0.0065816 & -2.723013753 \\
\hline
\end{tabular}

Table 4 Comparison of $\boldsymbol{y}_{S \boldsymbol{J}}^{*}$ of Salameh and Jaber (2000) and $\boldsymbol{y}_{\boldsymbol{M} \boldsymbol{J}}^{*}$ of Maddah and Jaber (2008)

\begin{tabular}{r|r|r|r|r|r}
\hline \multicolumn{1}{c|}{$b$} & $E[P]$ & \multicolumn{1}{c|}{$y_{S J}^{*}$} & \multicolumn{1}{c|}{$y_{M J}^{*}$} & \multicolumn{1}{c}{$\Delta_{4}$} & \multicolumn{2}{|c}{$\Delta_{4}(\%)$} \\
\hline 0.01 & 0.005 & 1419.28 & 1420.90 & 1.63 & 0.11 \\
\hline 0.04 & 0.020 & 1434.62 & 1441.26 & 6.65 & 0.46 \\
\hline 0.07 & 0.035 & 1450.16 & 1462.05 & 11.89 & 0.82 \\
\hline 0.10 & 0.050 & 1465.91 & 1483.28 & 17.37 & 1.18 \\
\hline 0.15 & 0.075 & 1492.61 & 1519.64 & 27.03 & 1.81 \\
\hline 0.20 & 0.100 & 1519.82 & 1557.23 & 37.41 & 2.46 \\
\hline 0.25 & 0.125 & 1547.51 & 1596.07 & 48.56 & 3.14 \\
\hline 0.30 & 0.150 & 1575.60 & 1636.13 & 60.53 & 3.84 \\
\hline 0.40 & 0.200 & 1632.70 & 1719.84 & 87.14 & 5.34 \\
\hline 0.50 & 0.250 & 1690.24 & 1807.94 & 117.71 & 6.96 \\
\hline
\end{tabular}




\section{Chapter 3 Observation 2-- A Note on Khan et al. (2011)}

In this chapter, a minor error in Khan et al. (2011) is identified. A revised model is presented and numerically compared to the model of Khan et al. (2011). A multi-factor sensitivity analysis is also provided. The different parts of the two models are labeled in boldfaced characters.

\subsection{Introduction}

\subsubsection{Review of Khan et al. (2011)}

Khan et al. (2011) extended the model of Salameh and Jaber (2000) by considering inspection errors. A mathematical model with closed form solution is developed. Numerical examples were also provided to illustrate the solution procedure. A similar modelling approach to Maddah and Jaber (2008) was used, i.e. renewal-reward theorem. Apart from the same notation in Salameh and Jaber (2000), the other notations in this paper are presented below:

\footnotetext{
$m_{1} \quad$ probability of Type I error (classifying a non-defective item as defective), a random variable

$m_{2} \quad$ probability of Type II error (classifying a defective item as nondefective), a random variable

$f\left(m_{1}\right) \quad$ probability density function of $m_{1}$

$f\left(m_{2}\right) \quad$ probability density function of $m_{2}$

$t_{1} \quad$ inspection time in a cycle

$t_{2}$ the remaining time in a cycle, after the defective items are screened
} 
out

$B_{1} \quad$ number of items that are classified as defective in one cycle ), a random variable

$B_{2} \quad$ number of defective items that are returned from the market in one cycle), a random variable

$c_{a} \quad$ cost of accepting a defective item

$c_{r} \quad$ cost of rejecting a non-defective item

$T \quad$ cycle length $\left(T=\frac{y(1-P)\left(1-m_{1}\right)}{D}\right)$, a random variable

$A$ a parameter used for simplifying the holding cost, a random variable, where $A=1-D / x-\left(m_{1}+P\right)+P\left(m_{1}+m_{2}\right)$

There are three assumptions in the paper:

1) The probability of misclassification errors is known.

2) The items that are returned from the market are stored with those that are classified as defective by the inspector. They are all sold as a single batch at the end of each cycle at a discounted price.

3) The number of non-defective items is at least equal to the adjusted demand.

The authors established the model as follows:

Total profit per cycle $T P(y)=$ total revenue per cycle - total cost per cycle $=$ (the revenue from selling the good items + the salvage of items that are classified as defective items + the salvage of defective items that are returned from the market) - (the procurement cost + the screening cost, which is the sum of the costs of (a) inspection and (b) misclassification + the holding cost, which include the costs of (a) non-defective lot, (b) defective lot and (c) returned lot) 


$$
\begin{aligned}
=s y(1-P)( & \left.-m_{1}\right)+\boldsymbol{s y P m _ { 2 }}+v y(1-P) m_{1}+v y P \\
& -\left[K+c y+d y+c_{r}(1-P) y m_{1}+c_{a} P y m_{2}\right. \\
& \left.+\frac{h}{2}\left\{\left(\frac{2}{x}-\frac{D}{x^{2}}+\frac{A^{2}}{D}\right) y^{2}+y P m_{2} T\right\}\right]
\end{aligned}
$$

where the cycle length is

$$
T=\frac{y(1-P)\left(1-m_{1}\right)}{D}
$$

Then Khan et al. (2011) derived the expected total profit per cycle as:

$$
\begin{aligned}
E[T P(y)]= & s y(1-E[P])\left(1-E\left[m_{1}\right]\right)+\boldsymbol{s y} \boldsymbol{E}[\boldsymbol{P}] \boldsymbol{E}\left[\boldsymbol{m}_{\mathbf{2}}\right] \\
& +v y(1-E[P]) E\left[m_{1}\right]+v y E[P]-K-c y-d y \\
& -c_{r} y(1-E[P]) E\left[m_{1}\right]-c_{a} y E[P] E\left[m_{2}\right] \\
& -\frac{h}{2}\left\{\left(\frac{2}{x}-\frac{D}{x^{2}}+\frac{E\left[A^{2}\right]}{D}\right) y^{2}+\boldsymbol{y} \boldsymbol{E}[\boldsymbol{P}] \boldsymbol{E}\left[\boldsymbol{m}_{\mathbf{2}}\right] \boldsymbol{E}[\boldsymbol{T}]\right\}
\end{aligned}
$$

Expected cycle length

$$
E[T]=\frac{y(1-E[P])\left(1-E\left[m_{1}\right]\right)}{D}
$$

The expected annual profit was obtained by using the same approach in Maddah and Jaber (2008) (renewal-reward theorem) as

$$
E[T P U(y)]=\frac{E[T P(y)]}{E[T]}
$$

Then the optimal order size is

$$
y *=\sqrt{\frac{2 K D}{h E[P] E\left[m_{2}\right](1-E[P])\left(1-E\left[m_{1}\right]\right)+h D\left(\frac{2}{x}-\frac{D}{x^{2}}+\frac{E\left[A^{2}\right]}{D}\right)}}
$$




\subsubsection{Remarks on Khan et al. (2011)}

In Eq. (3.1), $P, m_{1}, m_{2}$, and $T$ are random variables. All other parameters are constant. $P, m_{1}$, and $m_{2}$ are independent while $T$ is a function of $P$ and $m_{1}$. According to the Proposition 2.3*, in Ross (2009), P.49, there is an error in the last term of Eq. (3.3) because $T=\frac{y(1-P)\left(1-m_{1}\right)}{D}$ is relevant to $P$.

$$
\begin{gathered}
\because y E[P] E\left[m_{2}\right] E[T]=y E[P] E\left[m_{2}\right] \frac{y(1-E[P])\left(1-E\left[m_{1}\right]\right)}{D} \\
=\frac{y^{2}}{D} E[P](1-E[P])\left(1-E\left[m_{1}\right]\right) E\left[m_{2}\right] \\
E\left[y P m_{2} T\right]=E\left[y P m_{2} \frac{y(1-P)\left(1-m_{1}\right)}{D}\right] \\
=\frac{y^{2}}{D} E[P(1-P)]\left(1-E\left[m_{1}\right]\right) E\left[m_{2}\right]
\end{gathered}
$$

According to Ross (2009) (P.46-47)

$$
\begin{aligned}
& \operatorname{Var}(X)=E\left[(X-E[x])^{2}\right]=E\left[X^{2}\right]-(E[X])^{2} \\
& E[P(1-P)]=E\left[P-P^{2}\right]=E[P]-E\left[P^{2}\right]=E[P]-\operatorname{Var}[P]-(E[P])^{2} \\
& =E[P](1-E[P])-\operatorname{Var}[P] \\
& \therefore E[P(1-P)]=E[P](1-E[P])-\operatorname{Var}[P] \neq E[P](1-E[P]) \\
& \therefore E\left[y P m_{2} T\right] \neq y E[P] E\left[m_{2}\right] E[T]
\end{aligned}
$$

*Proposition 2.3, in Ross (2009), P.49 If $X$ and $Y$ are independent, then for any function $h$ and $g, E[g(x) \cdot h(y)]=E[g(x)] E[h(y)]$. 


$$
\begin{aligned}
\therefore E\left[y P m_{2} T\right]= & \left.\frac{y^{2}}{D} E[P(1-P)]\right]\left(1-E\left[m_{1}\right]\right) E\left[m_{2}\right] \\
& \left.=\frac{y^{2}}{D}(E[P](1-E[P])-\operatorname{Var}[P])\right]\left(1-E\left[m_{1}\right]\right) E\left[m_{2}\right]
\end{aligned}
$$

There is a need to pay attention to the calculation of $E\left[A^{2}\right]$ for the same reason indicated above.

The items returned from the market that are defective items sell for a unit for a price of $v$, including those wrongly classified as non-defective. Teng et al. (2013) suggested that the revenue from items returned from market that are defective items but classified as non-defective should be $v y P m_{2}$ instead of $s y P m_{2}$. When it comes to the derivation of the expected total profit, Teng et al. (2013) made the same

mistake. $T=\frac{y(1-P)\left(1-m_{1}\right)}{D}$ is relevant to $P$, which violates the condition of Proposition 2.3, in Ross (2009), P.49. So, the expressions of $E[T P U(y)]$ and $y^{*}$ in Khan et al., (2011) need to be re-derived.

\subsection{Revised Model}

According to Teng et al. (2013) the total revenue should be rewritten as

$$
\begin{aligned}
R & =s y(1-P)\left(1-m_{1}\right)+v y(1-P) m_{1}+v y P m_{2}+v y P\left(1-m_{2}\right) \\
& =s y(1-P)\left(1-m_{1}\right)+v y(1-P) m_{1}+v y P .
\end{aligned}
$$

So, the total profit per cycle should be rewritten as

$$
\begin{gathered}
T P(y)=s y(1-P)\left(1-m_{1}\right)+v y(1-P) m_{1}+v y P \\
-\left[K+c y+d y+c_{r}(1-P) y m_{1}+c_{a} P y m_{2}\right. \\
+\frac{h}{2}\left\{\left(\frac{2}{x}-\frac{D}{x^{2}}+\frac{A^{2}}{D}\right) y^{2}+y P m_{2} T\right\}
\end{gathered}
$$




$$
\begin{aligned}
\because E\left[y P m_{2} T\right] & \left.=\frac{y^{2}}{D} E[P(1-P)]\right]\left(1-E\left[m_{1}\right]\right) E\left[m_{2}\right] \\
& \left.=\frac{y^{2}}{D}(E[P](1-E[P])-\operatorname{Var}[P])\right]\left(1-E\left[m_{1}\right]\right) E\left[m_{2}\right]
\end{aligned}
$$

The expected total profit is

$$
\begin{aligned}
& E[T P(y)] \\
& =s y(1-E[P])\left(1-E\left[m_{1}\right]\right)+v y(1-E[P]) E\left[m_{1}\right]+v y E[P] \\
& -\left[K+c y+d y+c_{r}(1-E[P]) y E\left[m_{1}\right]+c_{a} E[P] y E\left[m_{2}\right]\right. \\
& +\frac{h}{2}\left(\left(\frac{2}{x}-\frac{D}{x^{2}}+\frac{E\left[A^{2}\right]}{D}\right) y^{2}\right. \\
& \left.\left.+\frac{\left.\boldsymbol{y}^{2}(\boldsymbol{E}[\boldsymbol{P}](\mathbf{1}-\boldsymbol{E}[\boldsymbol{P}])-\operatorname{Var}[\boldsymbol{P}])\right]\left(\mathbf{1}-\boldsymbol{E}\left[\boldsymbol{m}_{\mathbf{1}}\right]\right) \boldsymbol{E}\left[\boldsymbol{m}_{\mathbf{2}}\right]}{\boldsymbol{D}}\right\}\right]
\end{aligned}
$$

The expected cycle length is

$$
E[T]=\frac{y(1-E[P])\left(1-E\left[m_{1}\right]\right)}{D}
$$

So the expected annual profit or the expected profit per unit time would be

$$
E[T P U(y)]=\frac{E[T P(y)]}{E[T]}
$$

or 


$$
\begin{aligned}
& E[T P U(y)] \\
& =s D+\frac{v D E\left[m_{1}\right]}{\left(1-E\left[m_{1}\right]\right)}+\frac{v D E[P]}{(1-E[P])\left(1-E\left[m_{1}\right]\right)} \\
& -\frac{D\left[\frac{K}{y}+c+d+c_{r}(1-E[P]) E\left[m_{1}\right]+c_{a} E[P] E\left[m_{2}\right]+\frac{h}{2}\left\{\left(\frac{2}{x}-\frac{D}{x^{2}}+\frac{E\left[A^{2}\right]}{D}\right) y\right\}\right]}{(1-E[P])\left(1-E\left[m_{1}\right]\right)} \\
& -\frac{h y(E[P](1-E[P])-\operatorname{Var}[P])] E\left[m_{2}\right]}{2(1-E[P])}
\end{aligned}
$$

The first derivative of equation (3.10) is

$$
\begin{aligned}
E\left[T P U^{\prime}(y)\right]= & -\frac{D\left[-\frac{K}{y^{2}}+\frac{h}{2}\left(\frac{2}{x}-\frac{D}{x^{2}}+\frac{E\left[A^{2}\right]}{D}\right)\right]}{(1-E[P])\left(1-E\left[m_{1}\right]\right)} \\
& -\frac{h(E[P](1-E[P])-\operatorname{Var}[P])] E\left[m_{2}\right]}{2(1-E[P])}
\end{aligned}
$$

The second derivative of equation (3.10) is the same as in Khan et al. (2011):

$E\left[T P U^{\prime \prime}(y)\right]=-\frac{2 D K}{y^{3}(1-E[P])\left(1-E\left[m_{1}\right]\right)}$

By setting the first derivative equal to zero and solving for $y$, the new optimal order size is

$$
y^{*}=\frac{\sqrt{2 D K}}{\sqrt{h D\left(\frac{2}{x}-\frac{D}{x^{2}}+\frac{E\left[A^{2}\right]}{D}\right)+h(E[P](1-E[P])-\operatorname{Var}[\boldsymbol{P}]) E\left[m_{2}\right]\left(1-E\left[m_{1}\right]\right)}}
$$

Here

$$
A=1-D / x-\left(m_{1}+P\right)+P\left(m_{1}+m_{2}\right)
$$




$$
\begin{array}{rl}
A^{2}=1-2 P+ & P^{2}+\frac{D^{2}}{x^{2}}-\frac{2 D}{x}+\frac{2 D P}{x}-2 m_{1}+4 P m_{1}-2 P^{2} m_{1}+\frac{2 D m_{1}}{x} \\
& -\frac{2 D P m_{1}}{x}+m_{1}^{2}-2 P m_{1}^{2}+P^{2} m_{1}^{2}+2 P m_{2}-2 P^{2} m_{2}-\frac{2 D P m_{2}}{x} \\
& -2 P m_{1} m_{2}+2 P^{2} m_{1} m_{2}+P^{2} m_{2}^{2} \\
E\left[A^{2}\right]=1-2 & E[P]+E\left[P^{2}\right]+\frac{D^{2}}{x^{2}}-\frac{2 D}{x}+\frac{2 D E[P]}{x}-2 E\left[m_{1}\right]+4 E[P] E\left[m_{1}\right] \\
& -2 E\left[P^{2}\right] E\left[m_{1}\right]+\frac{2 D E\left[m_{1}\right]}{x}-\frac{2 D E[P] E\left[m_{1}\right]}{x}+E\left[m_{1}^{2}\right] \\
& -2 E[P] E\left[m_{1}^{2}\right]+E\left[P^{2}\right] E\left[m_{1}^{2}\right]+2 E[P] E\left[m_{2}\right]-2 E\left[P^{2}\right] E\left[m_{2}\right] \\
& -\frac{2 D E[P] E\left[m_{2}\right]}{x}-2 E[P] E\left[m_{1}\right] E\left[m_{2}\right]+2 E\left[P^{2}\right] E\left[m_{1}\right] E\left[m_{2}\right] \\
+ & E\left[P^{2}\right] E\left[m_{2}^{2}\right]
\end{array}
$$

If

$P \sim U(a, b)$

$E[P]=\frac{a+b}{2}$

$\operatorname{Var}[P]=\frac{(b-a)^{2}}{12}$

$E\left[P^{2}\right]=\int_{a}^{b} x^{2} \frac{1}{b-a} \mathrm{~d} x=\frac{1}{3}\left(a^{2}+a b+b^{2}\right)$

$E\left[m_{1}^{2}\right]$ and $E\left[m_{2}^{2}\right]$ can be calculated similarly.

\subsection{Numerical Comparison of the Model and Sensitivity Analysis}

\subsubsection{The Difference of $E[P(1-P)]$ and $E[P](1-E[P])$}

There is a minor error in the model of Khan et al. (2011) because the last term in the expression of the expected total profit Eq. (3.3) is $y E[P] E\left[m_{2}\right] E[T]$, which is not equal to $E\left[y P m_{2} T\right]$. The reason for $E\left[y P m_{2} T\right] \neq y E[P] E\left[m_{2}\right] E[T]$ is 
because $E[P(1-P)]=E[P](1-E[P])-\operatorname{Var}[P] \neq E[P](1-E[P])$. Table 5 shows the difference between $E[P(1-P)]$ and $E[P](1-E[P])$ for different values of $b$.

Here

$$
\begin{aligned}
\Delta_{5} & =E[P](1-E[P])-E[P(1-P)] \\
& =E[P](1-E[P])-(E[P](1-E[P])-\operatorname{Var}[P])=\operatorname{Var}[P]
\end{aligned}
$$

It can be seen from Table 5 that the difference of $E[P(1-P)]$ and $E[P](1-$ $E[P])$ is very minor but systematic. And the difference is increased when the volatility of $P$ is increased.

Table 5 The difference of $E[P(1-P)]$ and $E[P](1-E[P])$

\begin{tabular}{c|c|r|r|r}
\hline$b$ & $E[P]$ & $E[P](1-E[P])$ & $E[P(1-P)]$ & $\Delta_{5}=\operatorname{Var}[P]$ \\
\hline 0.01 & 0.005 & 0.047500 & 0.047492 & 0.000008 \\
\hline 0.04 & 0.020 & 0.019600 & 0.019467 & 0.000133 \\
\hline 0.07 & 0.035 & 0.033775 & 0.033367 & 0.000408 \\
\hline 0.10 & 0.050 & 0.047500 & 0.046667 & 0.000833 \\
\hline 0.15 & 0.075 & 0.069375 & 0.067500 & 0.001875 \\
\hline 0.20 & 0.100 & 0.090000 & 0.086667 & 0.003333 \\
\hline 0.25 & 0.125 & 0.109375 & 0.104167 & 0.005208 \\
\hline 0.30 & 0.150 & 0.127500 & 0.120000 & 0.007500 \\
\hline 0.40 & 0.200 & 0.160000 & 0.146667 & 0.013333 \\
\hline 0.50 & 0.250 & 0.187500 & 0.166667 & 0.020833 \\
\hline
\end{tabular}

\subsubsection{Numerical Comparison of Khan et al. (2011) to the Revised Model}

To compare the model of Khan et al. (2011) to the revised model, a numerical example is presented in this section. The values of the parameters are taken from Khan et al. (2011), which are shown below:

Let $D=50,000$ units/year, $c=\$ 25 /$ unit, $K=\$ 100 /$ cycle, $s=\$ 50 /$ unit, $d$ $=\$ 0.5 /$ unit,$\quad x=1$ unit $/ \mathrm{min}=175,200$ units $/$ year,$\quad h=\$ 5 /$ unit $/$ year, $v=\$ 20 /$ unit, 
$c_{a}=\$ 500 /$ unit, $c_{r}=\$ 100 /$ unit, $E\left[m_{1}\right]=0.02, E\left[m_{2}\right]=0.02$. And the fraction of imperfect quality items, $P$, uniformly distributed on $(a, b), 0<a<b<1$, i.e., $P \sim U(a, b) . a=0$. Table 6 shows the differences in the values of the optimal lot size $y^{*}$ and the expected total profit per unit of time $E[T P U(y)]$ for different values of $b$ for the two models. (Matlab code is shown in Appendix 2).

Table 6 Comparison of the optimal lot size $y^{*}$ and the expected total profit per unit time $E[T P U]$ between Khan et al. (2011) and revised model

\begin{tabular}{c|c|c|r|r|r|r|r}
\hline$b$ & $E[P]$ & $y_{\text {Khan }(201}^{*}$ & $y_{\text {revised }}^{*}$ & $\Delta_{6}(\%)$ & $E[T P U]_{\text {Khan }(2011}$ & $E[\text { TPU }]_{\text {revised }}$ & $\Delta_{7}(\%)$ \\
\hline 0.01 & 0.005 & 1439.33 & 1439.33 & 0.0000 & 1106504.27 & 1106247.88 & -0.0232 \\
\hline 0.04 & 0.020 & 1453.90 & 1453.91 & 0.0001 & 1095087.44 & 1094046.21 & -0.0951 \\
\hline 0.07 & 0.035 & 1468.50 & 1468.51 & 0.0004 & 1083314.34 & 1081463.86 & -0.1708 \\
\hline 0.10 & 0.050 & 1483.10 & 1483.12 & 0.0009 & 1071168.04 & 1068482.76 & -0.2507 \\
\hline 0.15 & 0.075 & 1507.40 & 1507.44 & 0.0021 & 1050045.47 & 1045908.68 & -0.3940 \\
\hline 0.20 & 0.100 & 1531.58 & 1531.64 & 0.0038 & 1027744.94 & 1022076.01 & -0.5516 \\
\hline 0.25 & 0.125 & 1555.53 & 1555.63 & 0.0062 & 1004165.27 & 996876.64 & -0.7258 \\
\hline 0.30 & 0.150 & 1579.15 & 1579.30 & 0.0092 & 979193.35 & 970189.75 & -0.9195 \\
\hline 0.40 & 0.200 & 1624.92 & 1625.20 & 0.0173 & 924549.66 & 911794.55 & -1.3796 \\
\hline 0.50 & 0.250 & 1667.87 & 1668.34 & 0.0284 & 862593.22 & 845586.41 & -1.9716 \\
\hline
\end{tabular}

Here

$\Delta_{6}(\%)=\frac{y_{\text {revised }}^{*}-y_{\text {Khan }(2011)}^{*}}{y_{\text {Khan }(2011)}^{*}} \times 100 \%$

and

$\Delta_{7}(\%)=\frac{E[T P U]_{\text {revised }}-E[T P U]_{\text {Khan }(2011)}}{E[T P U]_{\text {Khan }(2011)}} \times 100 \%$

It is clear that the optimal lot size of $y^{*}$ in the revised model is larger than that of Khan et al (2011), and the gaps increase when $b$ increases. The expected total profit per unit of time $E[T P U]$ of the revised model is less than that of Khan et al 
(2011). The reason for it is that the expression of the total profit $T P$ of the revised model has not the term of $s y \mathrm{Pm}_{2}$. And the gaps of the expected total profit per unit of time $E[T P U]$ of two models are increase with the increase of $b$.

\subsubsection{Numerical Comparison of $T P U_{K h a n(2011)}\left(y_{K h a n(2011)}^{*}\right)$ and TPU $U_{\text {Khan (2011) }}\left(y_{\text {revised }}^{*}\right)$}

To show the difference between the model of Khan et al (2011) and the revised model clearly, the following hypothesis is tested by using the same parameters from Khan et al (2011).

$$
\begin{aligned}
& H_{04}: T P U_{\text {Khan(2011) }}\left(y_{\text {Khan(2011) }}^{*}\right)=T P U_{\text {Khan (2011) }}\left(y_{\text {revised }}^{*}\right) \\
& H_{a 4}: T P U_{\text {Khan(2011) }}\left(y_{\text {Khan(2011) }}^{*}\right) \neq T P U_{\text {Khan (2011) }}\left(y_{\text {revised }}^{*}\right)
\end{aligned}
$$

To be comparable, the total profit expression used in Khan et al (2011) is chosen, i.e. Eq. (3.1).

A Matlab program, which is provided in Appendix 3, has the following steps:

Step 1: Calculate the optimal order size $y_{\text {Khan(2011) }}^{*}$ by using Eq. (3.6).

Step 2: Calculate the optimal order size $y_{\text {revised }}^{*}$ by using Eq. (3.11).

Step 3: Generate the random numbers $P \sim U(0, b), m_{1} \sim U(0,0.04)$, and $m_{2} \sim U(0,0.04)$. Calculate the annual total profit $T P U_{K h a n(2011)}($ $y_{\text {Khan(2011) }}^{*}$ ) by using Eq. (3.1) and Eq. (2.10).

Step 4: Use the same random numbers of $P, m_{1}$, and $m_{2}$ generated in step 3 and calculate the annual total profit $T P U_{K h a n(2011)}\left(y_{\text {revised }}^{*}\right)$ by using Eq. (3.1)and Eq.(2.10).

Step 5: Repeat step 3 and step 4 for $n=1000$ times.

Step 6: Test $T P U_{K h a n(2011)}\left(y_{\text {Khan(2011) }}^{*}\right)=T P U_{\text {Khan(2011) }}\left(y_{\text {revised }}^{*}\right)$ by using 
paired sample t-test.

In step 6, paired sample t-test is the same method described in section 2.3.3. The results are shown in Table 7.

Table 7 Numerical Comparison of TPU $U_{K h a n(2011)}\left(y_{K h a n(2011)}^{*}\right)$ and $T P U_{\text {Khan(2011) }}\left(y_{\text {revised }}^{*}\right)$

\begin{tabular}{|c|c|c|c|c|c|c|}
\hline$b$ & $E[P]$ & $\begin{array}{l}\left.\overline{T P U}_{\text {Khan(2011) }}\right) \\
\left(y_{\text {Khan (2011) }}^{*}\right)\end{array}$ & $\begin{array}{c}\overline{T P U}_{\text {Khan }(2011} \\
\left(y_{\text {revised }}^{*}\right)\end{array}$ & $\Delta_{8}$ & $\Delta_{9}(\%)$ & P_value \\
\hline 0.01 & 0.050 & 1051483.86 & 1051484.32 & 0.47 & 0.0000 & $7.84 \mathrm{E}-01$ \\
\hline 0.04 & 0.020 & 1059131.20 & 1059133.17 & 1.97 & 0.0002 & $2.65 \mathrm{E}-01$ \\
\hline 0.07 & 0.035 & 1044288.33 & 1044291.24 & 2.90 & 0.0003 & $9.64 \mathrm{E}-02$ \\
\hline 0.10 & 0.050 & 1054189.46 & 1054199.95 & 10.49 & 0.0010 & $1.14 \mathrm{E}-08$ \\
\hline 0.15 & 0.075 & 1041889.24 & 1041908.10 & 18.86 & 0.0018 & $3.40 \mathrm{E}-24$ \\
\hline 0.20 & 0.100 & 1057991.03 & 1058033.22 & 42.19 & 0.0040 & $1.12 \mathrm{E}-93$ \\
\hline 0.25 & 0.125 & 1044636.49 & 1044699.20 & 62.70 & 0.0060 & $1.83 \mathrm{E}-168$ \\
\hline 0.30 & 0.150 & 1059576.62 & 1059671.80 & 95.18 & 0.0090 & $9.58 \mathrm{E}-261$ \\
\hline 0.40 & 0.200 & 1058484.93 & 1058668.29 & 183.36 & 0.0173 & $0.00 E+00$ \\
\hline 0.50 & 0.250 & 1053495.26 & 1053793.32 & 298.06 & 0.0283 & $0.00 \mathrm{E}+00$ \\
\hline
\end{tabular}

Here

$$
\begin{aligned}
& \Delta_{8}=\overline{T P U}_{K h a n(2011)}\left(y_{\text {Khan(2011) }}^{*}\right)-\overline{T P U}_{\text {Khan (2011) }}\left(y_{\text {revised }}^{*}\right) \\
& \Delta_{9}(\%)=\frac{\overline{T P U}_{K h a n(2011)}\left(y_{\text {Khan(2011) }}^{*}\right)-\overline{T P U}_{\text {Khan (2011) }}\left(y_{\text {revised }}^{*}\right)}{\overline{T P U}_{K h a n(2011)}\left(y_{\text {Khan (2011) }}^{*}\right)} \times 100 \%
\end{aligned}
$$

It can be seen from Table 7 that the differences between $T P U_{\text {Khan(2011) }}\left(y_{\text {Khan(2011) }}^{*}\right)$ and $T P U_{\text {Khan(2011) }}\left(y_{\text {revised }}^{*}\right)$ are minor but significant. And the differences increase when $b$ increases.

In reality, $P, m_{1}, m_{2}$ may not necessarily follow the uniform distribution, as assumed in the work of Khan et al., they may follow the normal distribution or some 
unknown distribution. Fortunately, the expected total profit per unit of time $E[T P U(y)]$ and the optimal lot size $y^{*}$ are only relevant to the first and second moments of $P, m_{1}, m_{2}$, which can be estimated by samples. For example, if there is a sample $P_{1}, P_{2}, \ldots P_{n}$,

$\widehat{E[P]}=\bar{P}=\frac{\sum_{i=1}^{n} P_{i}}{n}$

$\widehat{E\left[P^{2}\right]}=\overline{P^{2}}=\frac{\sum_{i=1}^{n} P_{i}^{2}}{n}$

\subsubsection{Sensitivity Analysis}

Since the difference between the revised model and the model of Khan et al (2011) is very small, there is no need to redo a one-factor sensitivity analysis since it has been done by Khan et al (2011). However, if there are several factors that change simultaneously, then performing one-factor sensitivity analysis may not be accurate because of possible interaction effect between/among the factors.

Khan et al (2011) have analyzed the relationship between the expected annual profit and the fraction of defectives $E[P]$ by fixing the order size per cycle $y$ at 1439. Since the expected annual profit $E[T P U]$ is not a linear function of $E[P]$ , $y, D, m_{1}, m_{2}$,etc., but a complicated function including multiple terms, interaction of factors on the expected annual profit $E[T P U]$ may occur. What would happen if the order size per cycle $y$ is adjusted at the same time?

An example of two factors changing at the same time is shown in Figure 1. The relationship between the expected annual profit $E[T P U]$ and the expected fraction of defectives $E[P]$ is explained in two cases:

Case 1: The expected annual profit $E[T P U]$ is calculated by fixing the order 
size per cycle $y$ at 1454 , which is the optimal order size per cycle $y^{*}$ of $E[P]=0.02$ with the other parameters as given in Section 3.3.2. For this case, only one factor, the fraction of defectives $E[P]$, is changed.

Case 2: The expected annual profit $E[T P U]$ is calculated by using the optimal order size per cycle $y^{*}$. The other parameters are the same as given in Section 3.3.2. For this case, two factors, the fraction of defectives $E[P]$ and the order size per cycle $y$, are changed simultaneously.

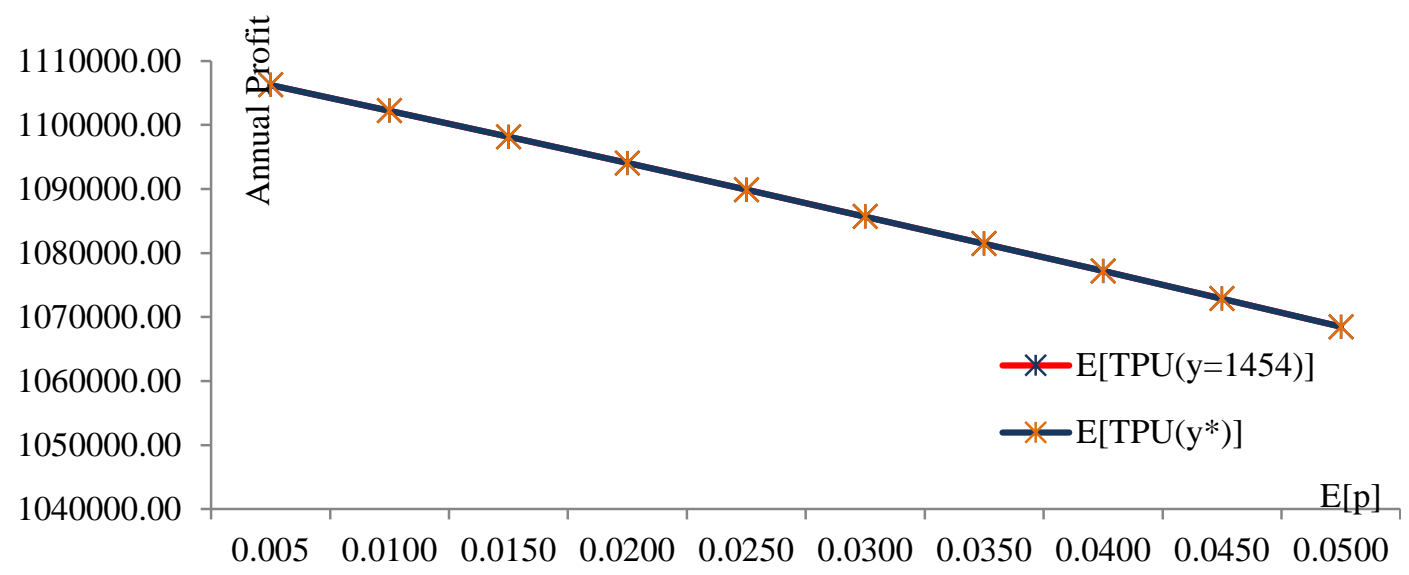

Figure 1 Comparison of $E[T P U(y=1454)]$ and $E\left[T P U\left(y^{*}\right)\right]$ for different $E[P]$

It can be seen from Figure 1 that the differences are insignificant such that one cannot distinguish between the two lines. Figure 2 shows the differences in the expected annual profit $E[T P U(y=1454)]-E\left[T P U\left(y^{*}\right)\right]$ for different values of $E[P]$. 


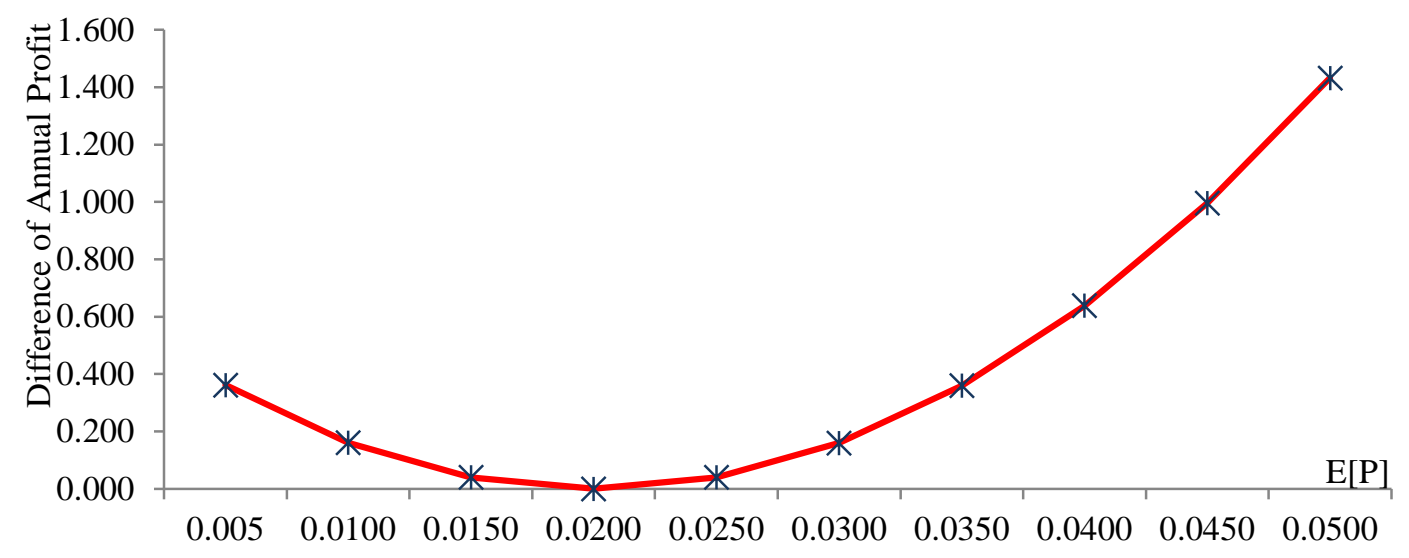

Figure 2 The difference of expected annual profits $E[T P U(y=1454)]$ and $E\left[T P U\left(y^{*}\right)\right]$ for different $E[P]$

Figure 2 shows that the fraction of defectives $E[P]$ and the order size per cycle $y$ have interaction effect on the expected annual profit $E[T P U]$. For this example, the interaction is not very noticeable, but it might be in other scenarios.

Table 8 shows the effects of changing one factor, while keeping the others unchanged and changing three factors simultaneously. A base comparison is provided where $E[T P U]=1094046.21, E[P]=0.02, D=50,000$, and $y=$ 1454. The other parameters are as given in Section 3.3.2.

The difference of the cumulative effects of changing one factor at a time and jointly changing three factors at the same time is

$91204.72-[-16864.79+109754.23+(-32.55)]$

$$
=91204.72-92856.89=-1652.17
$$


Table 8 The effects of only changing one factor at a time and the effect of changing three factors simultaneously.

\begin{tabular}{r|r|r|r|r}
\hline \multicolumn{1}{c|}{$D[P]$} & \multicolumn{1}{c|}{$y$} & \multicolumn{1}{c|}{$E[T P U]$} & \multicolumn{1}{c}{$\Delta_{E[T P U]}$} \\
\hline$+50 \%$ & 0 & 0 & 1077181.42 & -16864.79 \\
\hline 0 & $+10 \%$ & 0 & 1203800.44 & 109754.23 \\
\hline 0 & 0 & $+10 \%$ & 1094013.66 & -32.55 \\
\hline$+50 \%$ & $+10 \%$ & $+10 \%$ & 1185250.93 & 91204.72 \\
\hline
\end{tabular}

The results in Table 8 show that it is more meaningful to perform a multifactor sensitivity analysis.

For the example in Table 8, the expression of $E[T P U]$ is known. So, the interaction effect can be judged by its expression. If the exact expression is not known, a uniform experimental design and a regression model of the experimental results might be helpful (please refer to Li (2000) on this point). 


\section{Chapter 4 Observation 3-- A Note on Khan et al. (2014)}

Khan et al. (2014) contains similar errors as those found in Khan et al. (2011). In this chapter, the revised models are numerically compared to the model of Khan et al. (2014). For clarity, corrected/modified mathematical terms of the models are labeled in boldfaced characters.

\subsection{Review of Khan et al. (2014)}

Khan et al. (2014) constructed a model for an integrated supply chain taking into consideration quality inspection and learning in production. The notations used in Khan et al. (2014) are adopt here.

$n \quad$ number of shipments from the vendor to the buyer in a production cycle (a decision variable)

$Q \quad$ size of a shipment from the vendor to the buyer (a decision variable)

$T$ time between successive shipments(year), random variable

$d \quad$ buyer's unit screening cost (\$)

$x \quad$ buyer's screening rate (units/year)

$m_{1} \quad$ random variable (percentage) representing Type I error

$m_{2} \quad$ random variable (percentage) representing Type II error

$\gamma \quad$ percentage of defective items supplied by the vendor, random variable

$\gamma_{e} \quad$ percentage of defective items observed by the buyer through screening $\gamma_{e}=(1-\gamma) m_{1}+\gamma\left(1-m_{2}\right)$, random variable 
$f\left(m_{1}\right) \quad$ probability density function of $m_{1}$

$f\left(m_{2}\right) \quad$ probability density function of $m_{2}$

$f(\gamma) \quad$ probability density function of $\gamma$

D demand for the vendor (units/year)

$P \quad$ vendor's production rate (units/year)

c vendor's production cost per unit time (\$/year)

$b \quad$ vendor's learning exponent in the production process

$i \quad$ an index that represents vendors cycle of learning

F $\quad$ buyer’s fixed transportation cost per shipment (\$)

$v \quad$ buyer's transportation cost per unit of the product shipped (\$)

$A_{v} \quad$ vendor's fixed ordering or setup cost

$A_{b} \quad$ buy's setup cost

$h_{v} \quad$ vendor's unit holding cost for the product

$h_{b} \quad$ buy's unit holding cost for the product

$c_{a} \quad$ cost of falsely accepting a defective product

$c_{r} \quad$ cost of falsely rejecting a non-defective product

$M_{1} \quad$ a parameter used for simplifying the expected annual cost, a random variable, where $M_{1}=E[\gamma]$

$M_{2} \quad$ a parameter used for simplifying the expected annual cost, a random variable, where $M_{2}=1 /(1-E[\gamma])$

$M_{1 e} \quad$ a parameter used for simplifying the expected annual cost, a random variable, where $M_{1 e}=E\left[\gamma_{e}\right]$

$M_{2 e} \quad$ a parameter used for simplifying the expected annual cost, a random 
variable, where $M_{2 e}=1 /\left(1-E\left[\gamma_{e}\right]\right)$

The following notation are also used in the revised models.

$M_{3} \quad$ a parameter used for simplifying the expected annual cost, a random variable, where $M_{3}=E\left[(1-\gamma)^{2}\right]$

$M_{3 e} \quad$ a parameter used for simplifying the expected annual cost, a random variable, where $M_{3 e}=E\left[\left(1-\gamma_{e}\right)^{2}\right]$

\subsubsection{The Base Model}

The total cost of the two-level (vendor-buyer) supply chain in a cycle of Khan et al. (2014) is

$$
\begin{aligned}
T C=A_{v}+\frac{h_{v} n Q^{2}}{2 D} & \left\{(n-1)-(n-2) \frac{D}{P}\right\}+\frac{n c Q}{P}+A_{b} \\
& +n h_{b}\left\{\frac{Q(1-\gamma) T}{2}+\frac{\gamma Q^{2}}{x}\right\}+n d Q+n(F+v Q)
\end{aligned}
$$

Time between successive shipments (year) is

$$
T=\frac{(1-\gamma) Q}{D}
$$

After rearranging the terms, the expected total cost of the supply chain per cycle

$$
\begin{aligned}
E[T C(Q, n)]= & A_{v}+A_{b}+n F \\
& +\frac{n Q^{2}}{2 D}\left[\left\{h_{v}(n-1)-(n-2) \frac{D}{P}\right\}+\frac{2 h_{b} D E[\gamma]}{x}\right]+\frac{n c Q}{P} \\
& +\frac{\boldsymbol{n} \boldsymbol{h}_{\boldsymbol{b}} \boldsymbol{Q}(\mathbf{1}-\boldsymbol{E}[\boldsymbol{\gamma}]) \boldsymbol{E}[\boldsymbol{T}]}{\mathbf{2}}+n Q(d+v)
\end{aligned}
$$

and 


$$
E[T]=\frac{(1-E[\gamma]) Q}{D}
$$

Using $n E[T]$ as the total cycle time, the expected annual cost, by using the renewalreward theory as in Maddah and Jaber (2008), is

$$
\begin{aligned}
E[T C U(Q, n)] & =\frac{E T C(Q, n)}{n E[T]} \\
& =\frac{D}{(1-E[\gamma]) Q}\left\{\frac{\left(A_{v}+A_{b}\right)}{n}+F\right\}+\frac{(d+v) D}{(1-E[\gamma])} \\
& +\frac{Q}{2(1-E[\gamma])}\left[h_{v}\left\{(n-1)-(n-2) \frac{D}{P}\right\}+\frac{\left.2 h_{b} D E[\gamma]\right]}{x}\right] \\
& +\frac{c D}{P(1-E[\gamma])}+\frac{\boldsymbol{h}_{b} \boldsymbol{Q}(\mathbf{1}-\boldsymbol{E}[\gamma])}{\mathbf{2}}
\end{aligned}
$$

or

$$
\begin{aligned}
E[T C U(Q, n)] & =\frac{D}{(1-E[\gamma]) Q}\left\{\frac{\left(A_{v}+A_{b}\right)}{n}+F\right\}+\frac{D}{(1-E[\gamma])}\left(d+v+\frac{c}{P}\right) \\
& +\frac{Q}{2}\left[\frac{h_{v}}{(1-E[\gamma])}\left\{(n-1)-(n-2) \frac{D}{P}\right\}\right. \\
& \left.+\boldsymbol{h}_{\boldsymbol{b}}\left\{(\mathbf{1}-\boldsymbol{E}[\gamma])+\frac{2 D E[\gamma]}{x(1-E[\gamma])}\right\}\right]
\end{aligned}
$$

Let $M_{1}=E[\gamma]$ and $M_{2}=1 /(1-E[\gamma])$

$$
\begin{aligned}
E[T C U(Q, n)] & =\frac{D M_{2}}{Q}\left\{\frac{\left(A_{v}+A_{b}\right)}{n}+F\right\}+D M_{2}\left(d+v+\frac{c}{P}\right) \\
& +\frac{Q}{2}\left[h_{v} M_{2}\left\{(n-1)-(n-2) \frac{D}{P}\right\}\right. \\
& \left.+h_{b}\left\{\left(\mathbf{1}-\boldsymbol{M}_{\mathbf{1}}\right)+\frac{2 D M_{1} M_{2}}{x}\right\}\right]
\end{aligned}
$$




\subsubsection{The Model with Inspection Errors}

If taking inspection errors into consideration, the fraction of defective item and its expected value as a result of imperfect inspection is given by Khan et al. (2014) as

$\gamma_{e}=(1-\gamma) m_{1}+\gamma\left(1-m_{2}\right)$

and

$$
E\left[\gamma_{e}\right]=(1-E[\gamma]) E\left[m_{1}\right]+E[\gamma]\left(1-E\left[m_{2}\right]\right)
$$

The expected time interval between successive shipments is

$E[T]=\frac{\left(1-E\left[\gamma_{e}\right]\right) Q}{D}=\frac{\left\{E[\gamma] E\left[m_{1}\right]+(1-E[\gamma])\left(1-E\left[m_{2}\right]\right)\right\} Q}{D}$

The expected total annual cost is written as

$$
\begin{aligned}
E[T C U(Q, n)] & =\frac{D}{\left(1-E\left[\gamma_{e}\right]\right) Q}\left\{\frac{\left(A_{v}+A_{b}\right)}{n}+F\right\}+\frac{D}{\left(1-E\left[\gamma_{e}\right]\right)}\left(d+v+\frac{c}{P}\right) \\
& +\frac{Q}{2}\left[\frac{h_{v}}{\left(1-E\left[\gamma_{e}\right]\right)}\left\{(n-1)-(n-2) \frac{D}{P}\right\}\right. \\
& \left.+h_{b}\left\{\left(\mathbf{1}-\boldsymbol{E}\left[\gamma_{e}\right]\right)+\frac{2 D E\left[\gamma_{e}\right]}{x\left(1-E\left[\gamma_{e}\right]\right)}\right\}\right]+\frac{c_{a} E[\gamma] E\left[m_{2}\right] D}{\left(1-E\left[\gamma_{e}\right]\right)} \\
& +\frac{c_{r}(1-E[\gamma]) E\left[m_{1}\right] D}{\left(1-E\left[\gamma_{e}\right]\right)}
\end{aligned}
$$

Similarly, $M_{1 e}=E\left[\gamma_{e}\right]$ and $M_{2 e}=1 /\left(1-E\left[\gamma_{e}\right]\right)$ 


$$
\begin{aligned}
E[T C U(Q, n)] & =\frac{D M_{2 e}}{Q}\left\{\frac{\left(A_{v}+A_{b}\right)}{n}+F\right\}+D M_{2 e}\left(d+v+\frac{c}{P}\right) \\
& +\frac{Q}{2}\left[h_{v} M_{2 e}\left\{(n-1)-(n-2) \frac{D}{P}\right\}\right. \\
& \left.+h_{b}\left\{\left(\mathbf{1}-\boldsymbol{M}_{1}\right)+\frac{2 D M_{1 e} M_{2 e}}{x}\right\}\right]+D M_{2 e}\left\{c_{a} M_{1} E\left[m_{2}\right]\right. \\
& \left.+c_{r}\left(1-M_{1}\right) E\left[m_{1}\right]\right\}
\end{aligned}
$$

\subsubsection{The Model with Learning in Production}

When taking learning in production into consideration, the total cost of the two-level supply chain in Khan et al. (2014) is

$$
\begin{aligned}
T C_{i}(Q, n)=A_{v} & \\
& +\frac{h_{v} Q^{2-b}}{P(1-b)}\left[n\left[\{1+(i-1) n\}^{1-b}-\{(i-1) n\}^{1-b}\right]\right. \\
& -\frac{\left.n^{2-b}\left\{i^{1-b}-(i-1)^{1-b}\right\}\right]+\frac{h_{v} n(n-1) Q^{2}}{2 D}}{2-b} \\
& +\frac{c(n Q)^{1-b}\left\{i^{1-b}-(i-1)^{1-b}\right\}}{P(1-b)}+A_{b} \\
& +n h_{b}\left\{\frac{Q(1-\gamma) T}{2}+\frac{\gamma Q^{2}}{x}\right\}+n d Q+n(F+v Q)
\end{aligned}
$$

The expected total cost of Khan et al. (2014) is 


$$
\begin{aligned}
E\left[T C_{i}(Q, n)\right]= & A_{v}+A_{b}+n F \\
& +\frac{h_{v} Q^{2-b}}{P(1-b)}\left[n\left[\{1+(i-1) n\}^{1-b}-\{(i-1) n\}^{1-b}\right]\right. \\
& \left.-\frac{n^{2-b}\left\{i^{1-b}-(i-1)^{1-b}\right\}}{2-b}\right]+\frac{h_{v} n(n-1) Q^{2}}{2 D} \\
& +\frac{c(n Q)^{1-b}\left\{i^{1-b}-(i-1)^{1-b}\right\}}{P(1-b)} \\
& +n h_{b}\left\{\frac{\boldsymbol{Q}(\mathbf{1}-\boldsymbol{E}[\gamma]) \boldsymbol{E}[\boldsymbol{T}]}{\mathbf{2}}+\frac{E[\gamma] Q^{2}}{x}\right\}+n Q(d+v)
\end{aligned}
$$

where

$n E[T]=\frac{n(1-E[\gamma]) Q}{D}$

The expected annual cost is then given as

$$
\begin{aligned}
E\left[\operatorname{TCU}_{i}(Q, n)\right] & =\frac{D}{(1-E[\gamma]) Q}\left\{\frac{\left(A_{v}+A_{b}\right)}{n}+F\right\}+\frac{(d+v) D}{(1-E[\gamma])} \\
& +\frac{h_{v} D Q^{1-b}}{P(1-E[\gamma])(1-b)}\left[n\left[\{1+(i-1) n\}^{1-b}-\{(i-1) n\}^{1-b}\right]\right. \\
& \left.-\frac{n^{2-b}\left\{i^{1-b}-(i-1)^{1-b}\right\}}{2-b}\right]+\frac{h_{v}(n-1) Q}{2(1-E[\gamma])} \\
& +\frac{c(n Q)^{-b}\left\{i^{1-b}-(i-1)^{1-b}\right\}}{P(1-E[\gamma])(1-b)}+\frac{\boldsymbol{h}_{\boldsymbol{b}} \boldsymbol{Q}(\mathbf{1}-\boldsymbol{E}[\gamma])}{\mathbf{2}}+\frac{h_{b} E[\gamma] Q D}{x(1-E[\gamma])}
\end{aligned}
$$

Replacing $M_{1}=E[\gamma]$ and $M_{2}=1 /(1-E[\gamma])$ 


$$
\begin{aligned}
E\left[T C U_{i}(Q, n)\right] & =\frac{D M_{2}}{Q}\left\{\frac{\left(A_{v}+A_{b}\right)}{n}+F\right\}+(d+v) D M_{2}+\frac{h_{b} Q D M_{1} M_{2}}{x} \\
& +\frac{h_{v} D M_{2} Q^{1-b}}{P(1-b)}\left[n\left[\{1+(i-1) n\}^{1-b}-\{(i-1) n\}^{1-b}\right]\right. \\
& \left.-\frac{n^{2-b}\left\{i^{1-b}-(i-1)^{1-b}\right\}}{2-b}\right]+\frac{h_{v}(n-1) Q M_{2}}{2} \\
& +\frac{c M_{2}(n Q)^{-b}\left\{i^{1-b}-(i-1)^{1-b}\right\}}{P(1-b)}+\frac{\boldsymbol{h}_{\boldsymbol{b}} \boldsymbol{Q}\left(\mathbf{1}-\boldsymbol{M}_{\mathbf{1}}\right)}{\mathbf{2}}
\end{aligned}
$$

\subsection{Revised Model}

\subsubsection{The Revised Base Model}

For the same reason described in Section 3.1, i.e. $T$ is relevant to $\gamma$, the expression of the expected total cost $E[T C(Q, n)]$ and the expected annual total cost $E[T C U(Q, n)]$ need to be re-derived.

By substituting $T=\frac{(1-\gamma) Q}{D}$ into Eq. (4.1), we get

$$
\begin{aligned}
T C=A_{v}+\frac{h_{v} n Q^{2}}{2 D} & \left\{(n-1)-(n-2) \frac{D}{P}\right\}+\frac{n c Q}{P}+A_{b} \\
+ & n h_{b}\left\{\frac{Q(1-\gamma) T}{2}+\frac{\gamma Q^{2}}{x}\right\}+n d Q+n(F+v Q)
\end{aligned}
$$

Then,

$$
\begin{aligned}
T C=A_{v}+\frac{h_{v} n Q^{2}}{2 D}\left\{(n-1)-(n-2) \frac{D}{P}\right\}+\frac{n c Q}{P}+A_{b} \\
+n h_{b}\left\{\frac{Q^{2}(\mathbf{1}-\boldsymbol{\gamma})^{2}}{2 D}+\frac{\gamma Q^{2}}{x}\right\}+n d Q+n(F+v Q)
\end{aligned}
$$


and

$$
\begin{aligned}
E[T C(Q, n)]= & A_{v}+A_{b}+n F \\
& +\frac{n Q^{2}}{2 D}\left[\left\{h_{v}(n-1)-(n-2) \frac{D}{P}\right\}+\frac{\left.2 h_{b} D E[\gamma]\right]}{x}\right]+\frac{n c Q}{P} \\
& +\frac{\boldsymbol{n} \boldsymbol{h}_{b} \boldsymbol{Q}^{2} \boldsymbol{E}\left[(\mathbf{1}-\boldsymbol{\gamma})^{2}\right]}{\mathbf{2 D}}+n Q(d+v)
\end{aligned}
$$

and the expected cycle time is

$E[T]=\frac{(1-E[\gamma]) Q}{D}$

Using $n E[T]$ as the total cycle time, the expected annual cost, by using the renewalreward theory as in Maddah and Jaber (2008), would be

$$
\begin{aligned}
E[T C U(Q, n)] & =\frac{E T C(Q, n)}{n E[T]} \\
& =\frac{D}{(1-E[\gamma]) Q}\left\{\frac{\left(A_{v}+A_{b}\right)}{n}+F\right\}+\frac{(d+v) D}{(1-E[\gamma])} \\
& +\frac{Q}{2(1-E[\gamma])}\left[h_{v}\left\{(n-1)-(n-2) \frac{D}{P}\right\}+\frac{2 h_{b} D E[\gamma]}{x}\right] \\
& +\frac{c D}{P(1-E[\gamma])}+\frac{\boldsymbol{h}_{\boldsymbol{b}} \boldsymbol{Q E}\left[(\mathbf{1}-\boldsymbol{\gamma})^{2}\right]}{\mathbf{2}(\mathbf{1}-\boldsymbol{E}[\boldsymbol{\gamma}])}
\end{aligned}
$$

The difference between the revised model and Khan et al. (2014) is

$\frac{E\left[(1-\gamma)^{2}\right]}{(1-E[\gamma])} \neq(1-E[\gamma])$

The expected annual cost can also be rewritten as 


$$
\begin{aligned}
E[T C U(Q, n)] & =\frac{D}{(1-E[\gamma]) Q}\left\{\frac{\left(A_{v}+A_{b}\right)}{n}+F\right\}+\frac{D}{(1-E[\gamma])}\left(d+v+\frac{c}{P}\right) \\
& +\frac{Q}{2}\left[\frac{h_{v}}{(1-E[\gamma])}\left\{(n-1)-(n-2) \frac{D}{P}\right\}+\frac{\boldsymbol{h}_{\boldsymbol{b}} \boldsymbol{E}\left[(\mathbf{1}-\boldsymbol{\gamma})^{2}\right]}{(\mathbf{1}-\boldsymbol{E}[\boldsymbol{\gamma}])}\right. \\
& \left.+\frac{2 D E[\gamma]}{x(1-E[\gamma])}\right\}
\end{aligned}
$$

Let $M_{2}=1 /(1-E[\gamma])$ and $\boldsymbol{M}_{\mathbf{3}}=\boldsymbol{E}\left[(\mathbf{1}-\boldsymbol{\gamma})^{2}\right]$

$$
\begin{aligned}
E[T C U(Q, n)] & =\frac{D M_{2}}{Q}\left\{\frac{\left(A_{v}+A_{b}\right)}{n}+F\right\}+D M_{2}\left(d+v+\frac{c}{P}\right) \\
& +\frac{Q}{2}\left[h_{v} M_{2}\left\{(n-1)-(n-2) \frac{D}{P}\right\}\right. \\
& \left.+h_{b}\left(M_{2} M_{3}+\frac{2 D M_{1} M_{2}}{x}\right)\right]
\end{aligned}
$$

where

$M_{3}=E\left[(1-\gamma)^{2}\right]=\frac{1}{b-a} \int_{a}^{b}(1-\gamma)^{2} d \gamma=\frac{1}{3}\left(a^{2}+a b+b^{2}\right)+1-a-b$

The second derivative of Eq. (4.11) is the same as Khan et al. (2014). So, the optimal size of a shipment from the vendor to buyer $Q^{*}$ and the number of shipments per cycle $n^{*}$ can be obtained by using the same method described in Khan et al. (2014).

\subsubsection{The Revised Model with Inspection Errors}

The variable $T$ is also relevant to $\gamma_{e}$ for the expressions of expected total cost $E[T C(Q, n)]$ and the expected annual total cost $E[\operatorname{TCU}(Q, n)]$, which accounts for inspection errors, need to be re-derived.

Here

$\gamma_{e}=(1-\gamma) m_{1}+\gamma\left(1-m_{2}\right)$ 


$$
\begin{aligned}
& T C=A_{v}+\frac{h_{v} n Q^{2}}{2 D}\left\{(n-1)-(n-2) \frac{D}{P}\right\}+\frac{n c Q}{P}+A_{b}+n h_{b}\left\{\frac{Q\left(1-\gamma_{e}\right) T}{2}+\right. \\
& \left.\frac{\gamma_{e} Q^{2}}{x}\right\}+n d Q+n(F+v Q)+n Q(1-\gamma) C_{r} m_{1}+n Q \gamma C_{a} m_{2}
\end{aligned}
$$

by substituting the expression of $T$ below into Eq. (4.12)

$T=\frac{\left(1-\gamma_{e}\right) Q}{D}$

makes Eq. (4.12) to:

$T C=A_{v}+\frac{h_{v} n Q^{2}}{2 D}\left\{(n-1)-(n-2) \frac{D}{P}\right\}+\frac{n c Q}{P}+A_{b}+n h_{b}\left\{\frac{Q^{2}\left(1-\gamma_{e}\right)^{2}}{2 D}+\frac{\gamma_{e} Q^{2}}{x}\right\}+$

$n d Q+n(F+v Q)+n Q(1-\gamma) C_{r} m_{1}+n Q \gamma C_{a} m_{2}$

So, the expected total cost should be

$$
\begin{aligned}
E[T C(Q, n)]= & A_{v}+\frac{h_{v} n Q^{2}}{2 D}\left\{(n-1)-(n-2) \frac{D}{P}\right\}+\frac{n c Q}{P}+A_{b} \\
& +n h_{b}\left\{\frac{Q^{2} \boldsymbol{E}\left[\left(\mathbf{1}-\boldsymbol{\gamma}_{\boldsymbol{e}}\right)^{2}\right]}{2 \boldsymbol{D}}+\frac{E\left[\gamma_{e}\right] Q^{2}}{x}\right\}+n d Q \\
& +n(F+v Q) \\
& +n Q(1-E[\gamma]) C_{r} E\left[m_{1}\right]+n Q E[\gamma] C_{a} E\left[m_{2}\right]
\end{aligned}
$$

and

$E[T]=\frac{\left(1-E\left[\gamma_{e}\right]\right) Q}{D}$

Then, the expected annual cost is 


$$
\begin{aligned}
E[T C U(Q, n)] & =\frac{D}{\left(1-E\left[\gamma_{e}\right]\right) Q}\left\{\frac{\left(A_{v}+A_{b}\right)}{n}+F\right\}+\frac{D}{\left(1-E\left[\gamma_{e}\right]\right)}\left(d+v+\frac{c}{P}\right) \\
& +\frac{Q}{2}\left[\frac{h_{v}}{\left(1-E\left[\gamma_{e}\right]\right)}\left\{(n-1)-(n-2) \frac{D}{P}\right\}\right. \\
& \left.+h_{b}\left\{\frac{\boldsymbol{E}\left[\left(\mathbf{1}-\gamma_{e}\right)^{2}\right]}{\left(\mathbf{1}-\boldsymbol{E}\left[\gamma_{e}\right]\right)}+\frac{2 D E\left[\gamma_{e}\right]}{x\left(1-E\left[\gamma_{e}\right]\right)}\right\}\right]+\frac{c_{a} E[\gamma] E\left[m_{2}\right] D}{\left(1-E\left[\gamma_{e}\right]\right)} \\
& +\frac{c_{r}(1-E[\gamma]) E\left[m_{1}\right] D}{\left(1-E\left[\gamma_{e}\right]\right)}
\end{aligned}
$$

where

$$
E\left[\gamma_{e}\right]=(1-E[\gamma]) E\left[m_{1}\right]+E[\gamma]\left(1-E\left[m_{2}\right]\right)
$$

Similarly,

$$
\frac{E\left[\left(1-\gamma_{e}\right)^{2}\right]}{\left(1-E\left[\gamma_{e}\right]\right)} \neq\left(1-E\left[\gamma_{e}\right]\right)
$$

So, the revised model is different from the model of Khan et al. (2014).

Let $M_{1 e}=E\left[\gamma_{e}\right], M_{2 e}=1 /\left(1-E\left[\gamma_{e}\right]\right)$, and $\boldsymbol{M}_{\mathbf{3 e}}=\boldsymbol{E}\left[\left(\mathbf{1}-\boldsymbol{\gamma}_{\boldsymbol{e}}\right)^{2}\right]$, then

$$
\begin{aligned}
E[T C U(Q, n)] & =\frac{D M_{2 e}}{Q}\left\{\frac{\left(A_{v}+A_{b}\right)}{n}+F\right\}+D M_{2 e}\left(d+v+\frac{c}{P}\right) \\
& +\frac{Q}{2}\left[h_{v} M_{2 e}\left\{(n-1)-(n-2) \frac{D}{P}\right\}\right. \\
& \left.+h_{b}\left\{\boldsymbol{M}_{2 e} \boldsymbol{M}_{3 e}+\frac{2 D M_{1 e} M_{2 e}}{x}\right\}\right]+D M_{2 e}\left\{c_{a} M_{1} E\left[m_{2}\right]\right. \\
& +c_{r}\left(1-M_{1}\right) E\left[m_{1}\right]
\end{aligned}
$$

Since

$$
\begin{aligned}
\left(1-\gamma_{e}\right)^{2}= & \left(1-(1-\gamma) m_{1}-\gamma\left(1-m_{2}\right)\right)^{2} \\
& =1-2 \gamma+\gamma^{2}-2 m_{1}+4 \gamma m_{1}-2 \gamma^{2} m_{1}+m_{1}^{2}-2 \gamma m_{1}^{2}+\gamma^{2} m_{1}^{2} \\
& +2 \gamma m_{2}-2 \gamma^{2} m_{2}-2 \gamma m_{1} m_{2}+2 \gamma^{2} m_{1} m_{2}+\gamma^{2} m_{2}^{2}
\end{aligned}
$$




$$
\begin{aligned}
& M_{3 e}=E\left[\left(1-\gamma_{e}\right)^{2}\right]=E\left[\left(1-(1-\gamma) m_{1}-\gamma\left(1-m_{2}\right)\right)^{2}\right] \\
&=1-2 E[\gamma]+E\left[\gamma^{2}\right]-2 E\left[m_{1}\right]+4 E[\gamma] E\left[m_{1}\right]-2 E\left[\gamma^{2}\right] E\left[m_{1}\right] \\
&+ E\left[m_{1}^{2}\right]-2 E[\gamma] E\left[m_{1}^{2}\right]+E\left[\gamma^{2}\right] E\left[m_{1}^{2}\right]+2 E[\gamma] E\left[m_{2}\right] \\
&-2 E\left[\gamma^{2}\right] E\left[m_{2}\right]-2 E[\gamma] E\left[m_{1}\right] E\left[m_{2}\right]+2 E\left[\gamma^{2}\right] E\left[m_{1}\right] E\left[m_{2}\right] \\
&+E\left[\gamma^{2}\right] E\left[m_{2}^{2}\right]
\end{aligned}
$$

If $\gamma \sim U(a, b)$

$E[\gamma]=\frac{a+b}{2}$

$E\left[\gamma^{2}\right]=\int_{a}^{b} x^{2} \frac{1}{b-a} \mathrm{~d} x=\frac{1}{3}\left(a^{2}+a b+b^{2}\right)$

$E\left[m_{1}\right], E\left[m_{2}\right], E\left[m_{1}^{2}\right]$ and $E\left[m_{2}^{2}\right]$ can be calculated similarly.

The second derivative of Eq. (4.14) is the same as in Khan et al. (2014). So, the optimal size of a shipment from the vendor to buyer $Q^{*}$ and the number of shipments per cycle $n^{*}$ can be obtained by using the same method described in Khan et al. (2014).

\subsubsection{The Revised Model with Learning in Production}

Similar to Section 4.2.1, substituting $T=\frac{(1-\gamma) Q}{D}$ into Eq. (4.7) to get

$$
\begin{aligned}
T C_{i}(Q, n)=A_{v} & \\
& +\frac{h_{v} Q^{2-b}}{P(1-b)}\left[n\left[\{1+(i-1) n\}^{1-b}-\{(i-1) n\}^{1-b}\right]\right. \\
& \left.-\frac{n^{2-b}\left\{i^{1-b}-(i-1)^{1-b}\right\}}{2-b}\right]+\frac{h_{v} n(n-1) Q^{2}}{2 D} \\
& +\frac{c(n Q)^{1-b}\left\{i^{1-b}-(i-1)^{1-b}\right\}}{P(1-b)}+A_{b}+n h_{b}\left\{\frac{Q^{2}(\mathbf{1}-\boldsymbol{\gamma})^{2}}{2 \mathbf{D}}+\frac{\gamma Q^{2}}{x}\right\} \\
& +n d Q+n(F+v Q)
\end{aligned}
$$


Then the expected total cost is written as:

$$
\begin{aligned}
E\left[T C_{i}(Q, n)\right]= & A_{v}+A_{b}+n F \\
& +\frac{h_{v} Q^{2-b}}{P(1-b)}\left[n\left[\{1+(i-1) n\}^{1-b}-\{(i-1) n\}^{1-b}\right]\right. \\
& \left.-\frac{n^{2-b}\left\{i^{1-b}-(i-1)^{1-b}\right\}}{2-b}\right]+\frac{h_{v} n(n-1) Q^{2}}{2 D} \\
& +\frac{c(n Q)^{1-b}\left\{i^{1-b}-(i-1)^{1-b}\right\}}{P(1-b)} \\
& +n h_{b}\left\{\frac{\boldsymbol{n} Q^{2} E\left[(\mathbf{1}-\gamma)^{2}\right]}{\mathbf{2 D}}+\frac{E[\gamma] Q^{2}}{x}\right\}+n Q(d+v)
\end{aligned}
$$

and

$n E[T]=\frac{n(1-E[\gamma]) Q}{D}$

So the expected annual cost

$$
\begin{aligned}
E\left[T C U_{i}(Q, n)\right] & =\frac{D}{(1-E[\gamma]) Q}\left\{\frac{\left(A_{v}+A_{b}\right)}{n}+F\right\}+\frac{(d+v) D}{(1-E[\gamma])} \\
& +\frac{h_{v} D Q^{1-b}}{P(1-E[\gamma])(1-b)}\left[n\left[\{1+(i-1) n\}^{1-b}-\{(i-1) n\}^{1-b}\right]\right. \\
& \left.-\frac{n^{2-b}\left\{i^{1-b}-(i-1)^{1-b}\right\}}{2-b}\right]+\frac{h_{v}(n-1) Q}{2(1-E[\gamma])} \\
& +\frac{c(n Q)^{-b}\left\{i^{1-b}-(i-1)^{1-b}\right\}}{P(1-E[\gamma])(1-b)}+\frac{\boldsymbol{h}_{\boldsymbol{b}} \boldsymbol{Q E}\left[(\mathbf{1}-\boldsymbol{\gamma})^{2}\right]}{\mathbf{2}(\mathbf{1}-\boldsymbol{E}[\boldsymbol{\gamma}])}+\frac{h_{b} E[\gamma] Q D}{x(1-E[\gamma])}
\end{aligned}
$$

Let $M_{1}=E[\gamma], M_{2}=1 /(1-E[\gamma])$ and $\boldsymbol{M}_{\mathbf{3}}=\boldsymbol{E}\left[(\mathbf{1}-\boldsymbol{\gamma})^{\mathbf{2}}\right]$ 


$$
\begin{aligned}
E\left[T C U_{i}(Q, n)\right] & =\frac{D M_{2}}{Q}\left\{\frac{\left(A_{v}+A_{b}\right)}{n}+F\right\}+(d+v) D M_{2}+\frac{h_{b} Q D M_{1} M_{2}}{x} \\
& +\frac{h_{v} D M_{2} Q^{1-b}}{P(1-b)}\left[n\left[\{1+(i-1) n\}^{1-b}-\{(i-1) n\}^{1-b}\right]\right. \\
& \left.-\frac{n^{2-b}\left\{i^{1-b}-(i-1)^{1-b}\right\}}{2-b}\right]+\frac{h_{v}(n-1) Q M_{2}}{2} \\
& +\frac{c M_{2}(n Q)^{-b}\left\{i^{1-b}-(i-1)^{1-b}\right\}}{P(1-b)}+\frac{\boldsymbol{h}_{\boldsymbol{b}} \mathbf{Q} \boldsymbol{M}_{\mathbf{2}} \boldsymbol{M}_{\mathbf{3}}}{\mathbf{2}}
\end{aligned}
$$

The optimal size of a shipment from the vendor to buyer $Q^{*}$ and the number of shipments per cycle $n^{*}$ can be obtained by using the same method described in Khan et al. (2014).

\subsection{Numerical Example (Comparison)}

Numerical comparisons between the models of Khan et al. (2014) and the revised models of this chapter are given by using the same values of the parameters of Khan et al. (2014), which are shown below:

$D=1000$ units/year, $P=3200$ units/year, $c=\$ 100,000 /$ year, $x=1$ unit $/$ $\min =175,200$ units $/$ year,$A_{v}=\$ 400 /$ cycle, $A_{b}=\$ 25 /$ cycle, $c_{a}=\$ 200 /$ unit, $c_{r}=\$ 50 /$ unit, $h_{v}=\$ 5 /$ unit/year, $h_{b}=\$ 5 /$ unit/year, $d=\$ 0.5 /$ unit, $F=\$ 25 /$ shipment, $v$ $=\$ 2 /$ unit, $b=0.32$. Matlab codes for the numerical example can be seen in Appendix 4.

Table 9 shows the following results: $E[\gamma]=0.02, E\left[m_{1}\right]=0.02$, and $E\left[m_{2}\right]=0.02$.Table 10 shows the following results: $E[\gamma]=0.2, E\left[m_{1}\right]=0.2$, and $E\left[m_{2}\right]=0.2$, and when $E[\gamma]=0.5, E\left[m_{1}\right]=0.5$, and $E\left[m_{2}\right]=0.5$ the results are shown in Table 11. Here scenario 1, 2, 3, and 4 are the same as described in Khan 
et al. (2014), which are summarized below:

Scenario 1: a base model where there are no inspection errors and learning in production.

Scenario 2: the errors in inspection are involved, whereas there is no learning in production.

Scenario 3: learning in production is considered while inspection errors are assumed to be negligible.

Scenario 4: both inspection errors and learning in production are considered.

Table 9 Numerical comparison of Khan et al. (2014) and revised model $\left(E[\gamma]=0.02, E\left[m_{1}\right]=0.02\right.$, and $\left.E\left[m_{2}\right]=0.02\right)$

\begin{tabular}{c|c|c|c|c|c|c}
\hline \multirow{2}{*}{ Scenario } & \multicolumn{3}{|c|}{$\begin{array}{c}\text { Results of Model of Khan et } \\
\text { al.(2014) }\end{array}$} & \multicolumn{3}{c}{ Results of Revised Model } \\
\cline { 2 - 7 } & $n$ & $Q$ & $E[T C U(Q, n)]$ & $n$ & $Q$ & $E[T C U(Q, n)]$ \\
\hline 1 & 5 & 113.58 & 36415.23 & 5 & 113.58 & 36415.27 \\
\hline 2 & 4 & 136.36 & 38233.85 & 4 & 136.35 & 38233.94 \\
\hline 3 & 2 & 232.65 & 4636.62 & 2 & 232.64 & 4636.70 \\
\hline 4 & 2 & 233.89 & 5821.24 & 2 & 235.12 & 5810.13 \\
\hline
\end{tabular}

Table 10 Numerical comparison of Khan et al. (2014) and revised model $\left(E[\gamma]=0.2, E\left[m_{1}\right]=0.2\right.$, and $\left.E\left[m_{2}\right]=0.2\right)$

\begin{tabular}{c|r|r|r|r|r|r}
\hline Scenario & \multicolumn{3}{|c|}{$\begin{array}{c}\text { Results of Model of Khan et } \\
\text { al.(2014) }\end{array}$} & \multicolumn{3}{c}{ Results of Revised Model } \\
\cline { 2 - 7 } & $n$ & $Q$ & $E[T C U(Q, n)]$ & $n$ & $Q$ & $E[T C U(Q, n)]$ \\
\hline 1 & 3 & 182.93 & 44465.27 & 3 & 182.32 & 44472.88 \\
\hline 2 & 2 & 273.93 & 75711.82 & 2 & 272.40 & 75726.10 \\
\hline 3 & 2 & 257.10 & 5436.86 & 2 & 255.92 & 5447.55 \\
\hline 4 & 2 & 266.00 & 29834.75 & 2 & 272.93 & 29768.01 \\
\hline
\end{tabular}

It can be seen that the differences of the two models are not that significant, but they are increased when the volatility is increased. 
Table 11 Numerical comparison of Khan et al. (2014) and revised model $\left(E[\gamma]=0.5, E\left[m_{1}\right]=0.5\right.$, and $\left.E\left[m_{2}\right]=0.5\right)$

\begin{tabular}{c|r|r|r|r|r|r}
\hline Scenario & \multicolumn{3}{|c|}{$\begin{array}{c}\text { Results of Model of Khan et } \\
\text { al.(2014) }\end{array}$} & \multicolumn{3}{c}{ Results of Revised Model } \\
\cline { 2 - 7 } & $n$ & $Q$ & $E[T C U(Q, n)]$ & $n$ & $Q$ & $E[T C U(Q, n)]$ \\
\hline 1 & 1 & 596.60 & 70517.07 & 1 & 552.79 & 70756.18 \\
\hline 2 & 1 & 596.60 & 195517.07 & 1 & 566.31 & 195678.48 \\
\hline 3 & 2 & 266.00 & 8163.23 & 2 & 289.40 & 8286.12 \\
\hline 4 & 2 & 300.65 & 133163.23 & 2 & 293.01 & 133245.67 \\
\hline
\end{tabular}

It is worth mentioning that the costs of misclassification errors are not included in the Eq. (4.8). If both inspection errors and learning in production are considered, i.e. Scenario 4 in Khan et al. (2014), the expected annual cost is

$$
\begin{aligned}
E\left[T C U_{i}(Q, n)\right] & =\frac{D M_{2}}{Q}\left\{\frac{\left(A_{v}+A_{b}\right)}{n}+F\right\}+(d+v) D M_{2}+\frac{h_{b} Q D M_{1} M_{2}}{x} \\
& +\frac{h_{v} D M_{2} Q^{1-b}}{P(1-b)}\left[n\left[\{1+(i-1) n\}^{1-b}-\{(i-1) n\}^{1-b}\right]\right. \\
& \left.-\frac{n^{2-b}\left\{i^{1-b}-(i-1)^{1-b}\right\}}{2-b}\right]+\frac{h_{v}(n-1) Q M_{2}}{2} \\
& +\frac{c M_{2}(n Q)^{-b}\left\{i^{1-b}-(i-1)^{1-b}\right\}}{P(1-b)}+\frac{\boldsymbol{h}_{\boldsymbol{b}} \boldsymbol{Q}\left(\mathbf{1}-\boldsymbol{M}_{\mathbf{1}}\right)}{\mathbf{2}} \\
& +D M_{2 e}\left\{c_{a} M_{1} E\left[m_{2}\right]+c_{r}\left(1-M_{1}\right) E\left[m_{1}\right]\right\}
\end{aligned}
$$

Eq. (4.17) is not indicated in Khan et al. (2014). And the expected annual cost for Scenario 4 in the revised model is 


$$
\begin{aligned}
E\left[T C U_{i}(Q, n)\right] & =\frac{D M_{2 e}}{Q}\left\{\frac{\left(A_{v}+A_{b}\right)}{n}+F\right\}+(d+v) D M_{2 e} \\
& +\frac{h_{b} Q D M_{1 e} M_{2 e}}{x} \\
& +\frac{h_{v} D M_{2 e} Q^{1-b}}{P(1-b)}\left[n\left[\{1+(i-1) n\}^{1-b}-\{(i-1) n\}^{1-b}\right]\right. \\
& -\frac{\left.n^{2-b}\left\{i^{1-b}-(i-1)^{1-b}\right\}\right]+\frac{h_{v}(n-1) Q M_{2 e}}{2-b}}{2} \\
& +\frac{c M_{2 e}(n Q)^{-b}\left\{i^{1-b}-(i-1)^{1-b}\right\}}{P(1-b)}+\frac{\boldsymbol{h}_{b} Q \boldsymbol{M}_{2 e} \boldsymbol{M}_{3 e}}{2} \\
& +D M_{2 e}\left\{c_{a} M_{1} E\left[m_{2}\right]+c_{r}\left(1-M_{1}\right) E\left[m_{1}\right]\right\}
\end{aligned}
$$

Here, $\boldsymbol{M}_{2 e}=1 /\left(1-E\left[\gamma_{e}\right]\right)$, and $\boldsymbol{M}_{\mathbf{3} \boldsymbol{e}}=\boldsymbol{E}\left[\left(\mathbf{1}-\boldsymbol{\gamma}_{\boldsymbol{e}}\right)^{\mathbf{2}}\right]$. 


\section{Chapter 5 Conclusion}

In conclusion, this report presents three observations of the economic production quantity model with imperfect quality items with/without inspection.

The remarks and comparisons of the models of Salameh and Jaber (2000) and Maddah and Jaber (2008) are presented in the first observation. In this observation, the definition of the total profit per unit time in a finite time $t, T P U(t)$ is emphasized. The relationships of the objective functions of Salameh and Jaber (2000) and Maddah and Jaber (2008), and the expected total profit per unit time, $E[T P U]$ are identified. It is concluded that both models are not very accurate, but capable through the derivation, the numerical simulation and statistical comparison. The results show that the model of Maddah and Jaber (2008) is more accurate when the volatility of the percentage of defective items $P$ is small, which is more practical in reality.

In the second observation, a minor error in Khan et al. (2011) is first identified. Then, the revised model and numerical comparison are provided. The expression of the total profit per cycle is modified according to Teng et al. (2013). The condition of the properties of the expectation of random variables, i.e. the independence of random variables, is emphasized in the revised model. Numerical analysis shows that the difference of the optimal lot size $y^{*}$ between the models of Khan et al. (2011) and revised model increases when the volatility of the percentage of defective items $P$ increases. The same pattern is shown on the expected total profit per unit of time $E[T P U]$. By substituting $y_{\text {Khan(2011) }}^{*}$ and $y_{\text {revised }}^{*}$ into the total profit per unit of time, $T P U$, of Khan et al. (2011) respectively, the numerical 
comparison shows that the differences are minor but significant, which means that the differences are systematic.

The results of a multi-factor sensitivity analysis have been presented. When several factors are changed simultaneously, performing a multi-factor sensitivity analysis may be more accurate than performing one-factor sensitivity analysis because of possible interaction effect between/among the factors.

The same errors that were identified in Khan et al. (2011) are found in Khan et al. (2014). So, the third observation is the correction of the models of Khan et al. (2014). Numerical comparison shows that the differences of the two models are not that significant, but they are increased when the volatility is increased. 


\section{Appendices}

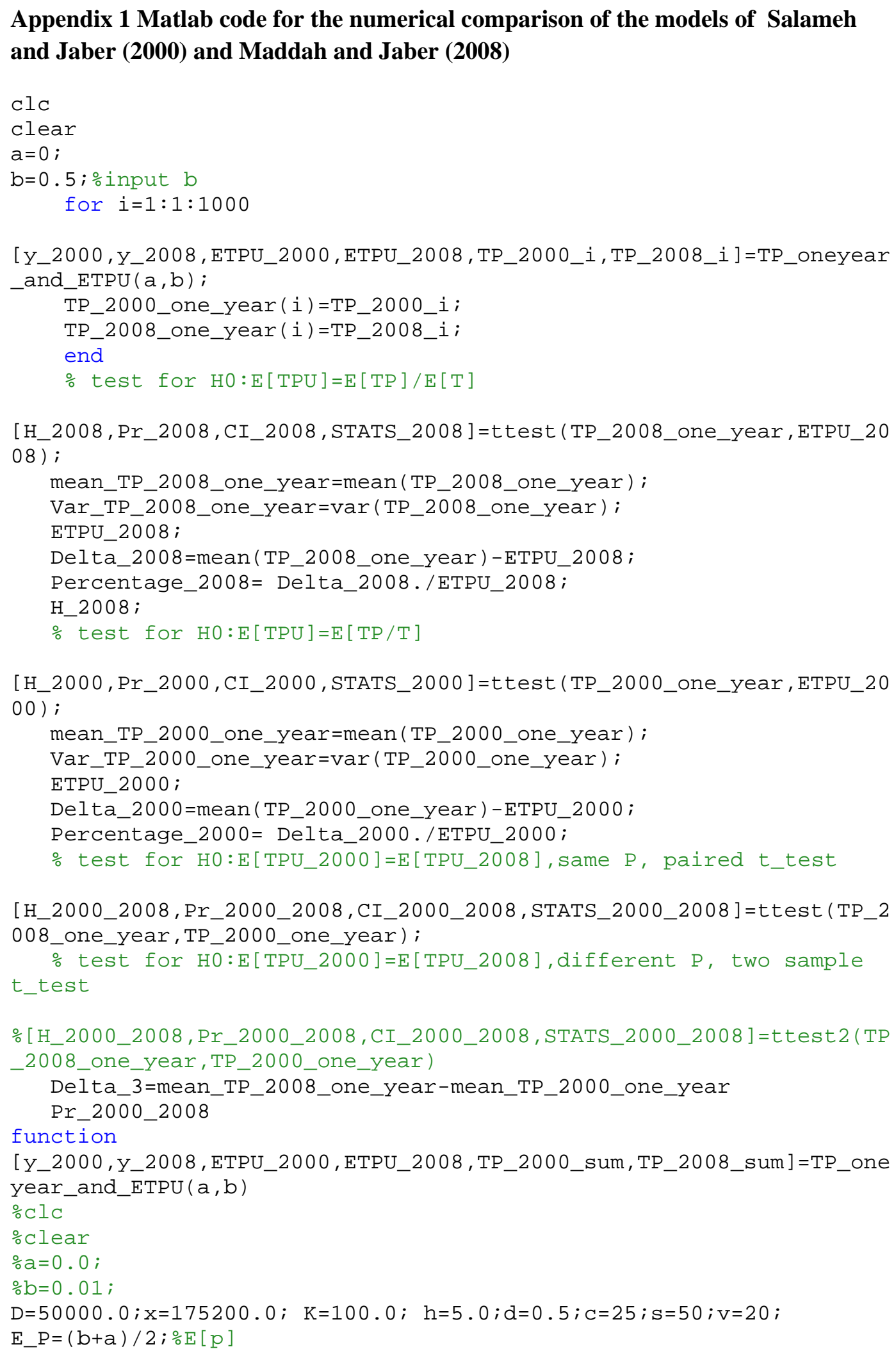




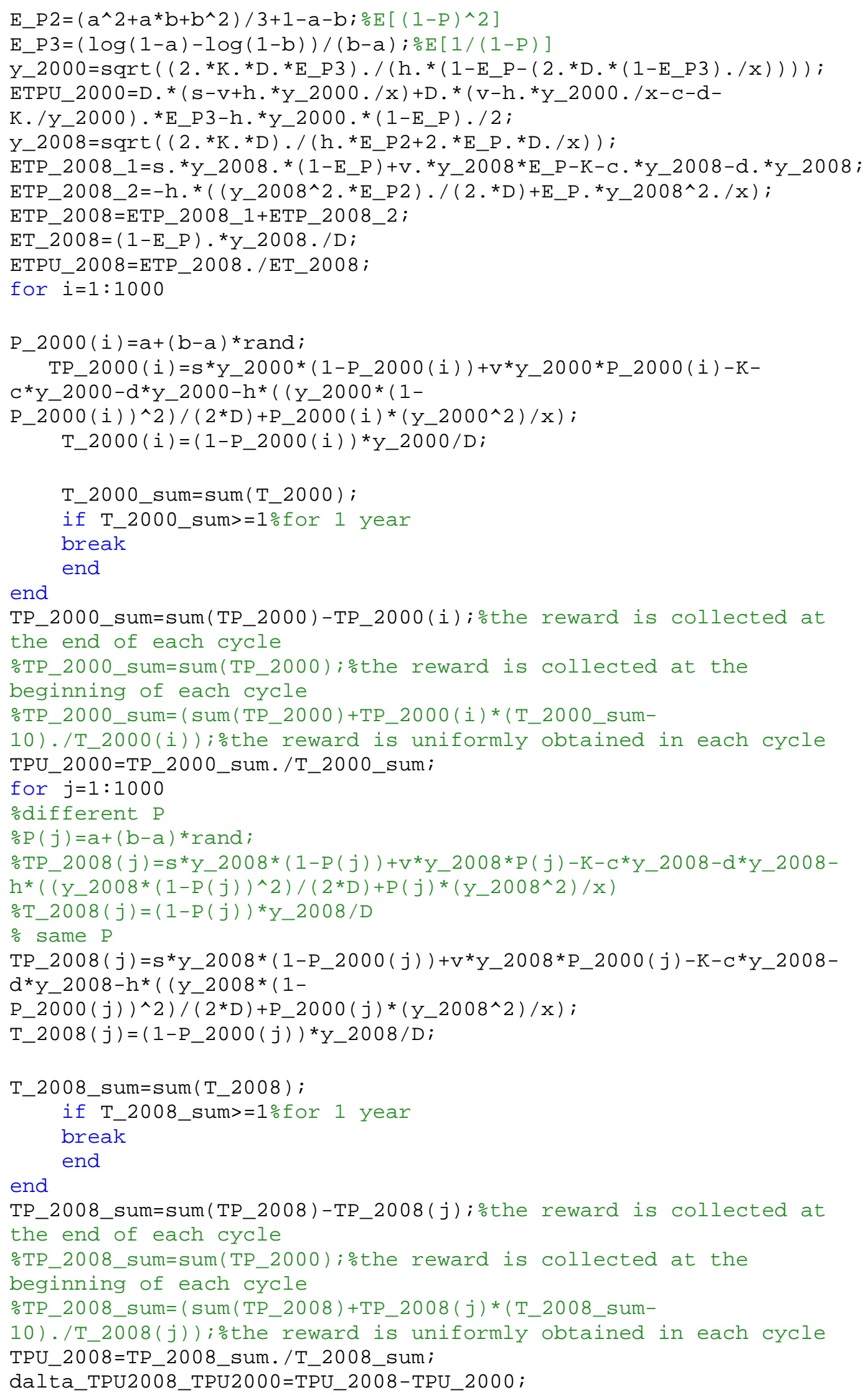




\section{Appendix 2 Matlab code for comparison of the optimal lot size and the expected total profit per unit time between Khan et al. (2011) and revised model}

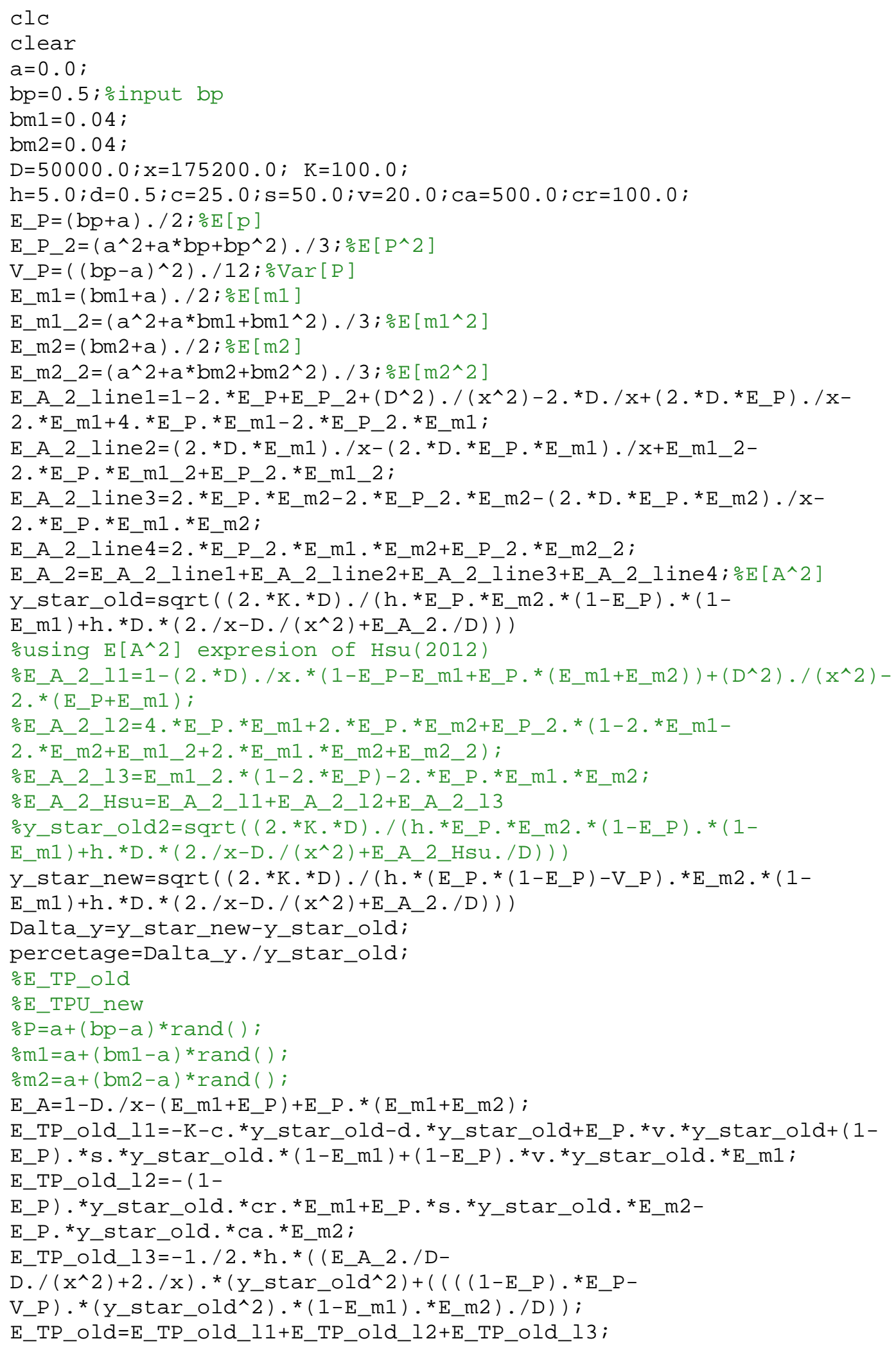


E_TP_new_l1=-K-C. ${ }^{*} y \_s t a r \_n e w-d .{ }^{*} y \_s t a r \_n e w+E \_P . *{ }^{*} \cdot{ }^{*} y \_s t a r \_n e w+(1-$

E_P $) \cdot{ }^{*} s \cdot{ }^{*} y \_s t a r \_n e w .{ }^{*}\left(1-\bar{E} \_m 1\right)+\left(1-E \_P\right) \cdot{ }^{*} V .{ }^{*} y \_s t a r \_n e w .{ }^{*} E \_m 1$;

E_TP_new_12=-(1-E_P $)$. ${ }^{*}$ y_star_new. ${ }^{*} \mathrm{cr} .{ }^{*} \mathrm{E} \_\mathrm{m} 1-$

E_P. ${ }^{*}$ y_star_new . ${ }^{*} \mathrm{Ca} .{ }^{*} \mathrm{E} \_\mathrm{m} 2$;

E_TP_new_13=-1./2.*h.* ( $E \_A \_2 . / D-$

$\left.D . /\left(x^{\wedge} 2\right)+2 . / x\right) . *\left(y \_s t a r \_n e w^{\wedge} 2\right)+\left(\left(\left(\left(1-E \_P\right) .{ }^{*} E \_P-\right.\right.\right.$

V_P $) . *\left(\right.$ Y_star_new^2).* $\left.\left.\left.\left(\overline{1}-E \_m 1\right) .{ }^{*} E \_m 2\right) . / D\right)\right)$;

E_TP_new=E_TP_new_l1+E_TP_new_12+E_TP_new_13;

E_Dalta_TP=E_TP_new-E_TP_old;

E_T_old=(1-E_P ). ${ }^{*}$ y_star_old. * $\left(1-E \_m 1\right) . / D$;

E_T_new $=\left(1-E \_P\right) .{ }^{*}$ y_star_new. ${ }^{*}\left(1-E \_m 1\right) . / D$;

E_TPU_old=E_TP_old./E_T_old;

E_TPU_new=E_TP_new./E_T_new;

Dalta_E_TPU=E_TPU_new-E_TPU_old;

Percentage_Dalta_E_TPU=Dalta_E_TPU./E_TPU_old; 


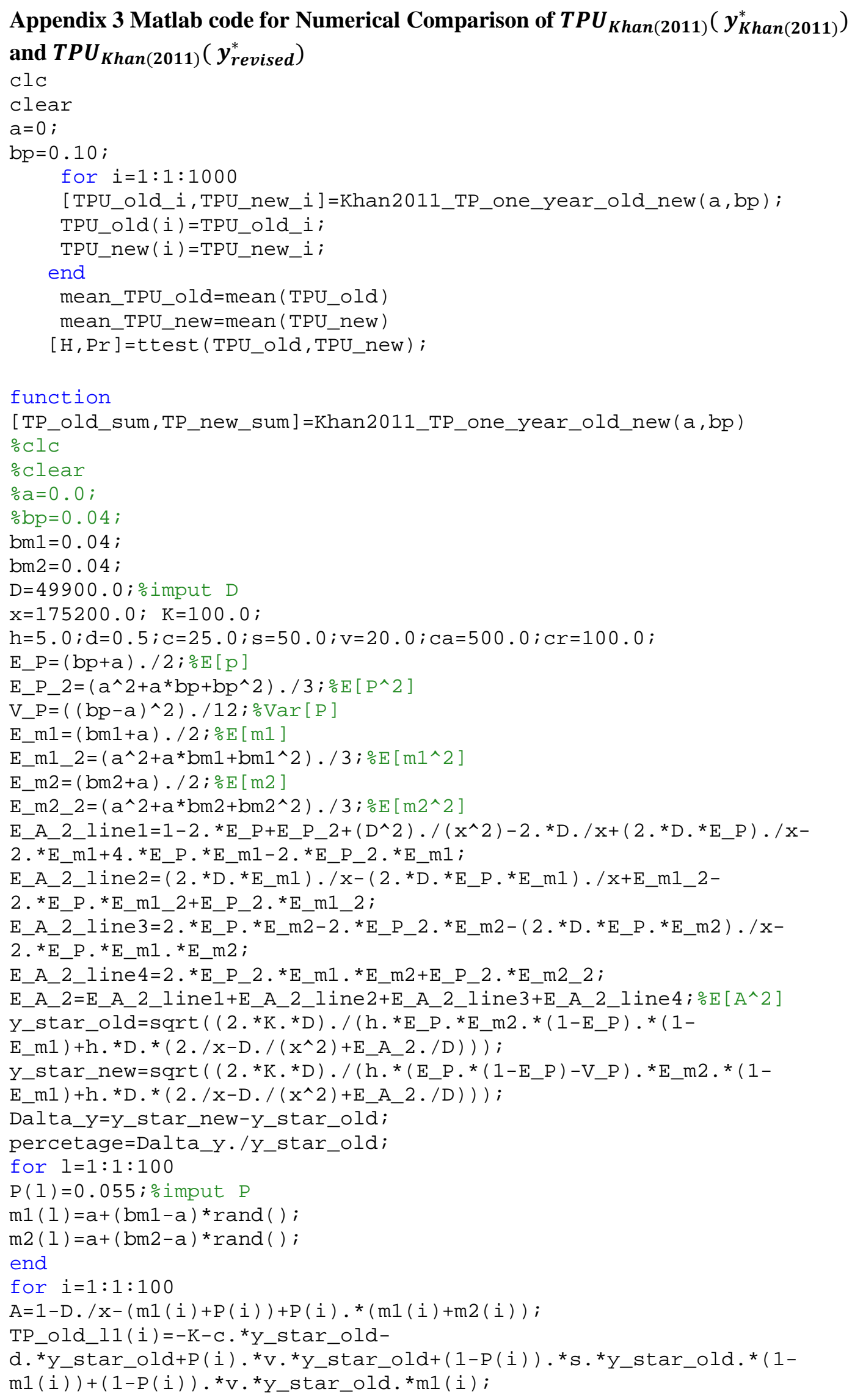


TP_old_12(i) $=-(1-$

$\mathrm{P}(\overline{\mathrm{i}})) \cdot{ }^{*} \mathrm{y}$ star_old. ${ }^{*} \mathrm{cr} .{ }^{*} \mathrm{~m} 1(\mathrm{i})+\mathrm{P}(\mathrm{i}) \cdot{ }^{*} \mathrm{~s} .{ }^{*} \mathrm{y}$ star_old. ${ }^{*} \mathrm{~m} 2(\mathrm{i})-$

$\mathrm{P}(\mathrm{i}) .{ }^{*} \mathrm{y}$ star_old. ${ }^{*} \mathrm{ca} \cdot{ }^{*} \mathrm{~m} 2(\mathrm{i})$;

TP_old_13 $(i)=-1 . / 2 .{ }^{*} \mathrm{~h} .{ }^{*}\left(\left(\mathrm{~A}^{\wedge} 2 . / \mathrm{D}-\right.\right.$

D. $\left./\left(x^{\wedge} 2\right)+2 . / x\right) . *\left(y \_s t a r \_o l d \wedge 2\right)+(((1-$

$\left.\left.\left.P(i)) .{ }^{*} P(i) .{ }^{*}\left(y \_s t a r \_o l d^{\wedge} 2\right) .{ }^{*}(1-m 1(i)) .{ }^{*} m 2(i)\right) . / D\right)\right)$;

TP_old $(i)=T P \_0 l d \_11(i)+T P \_0 l d \_12(i)+T P \_0 l d \_13(i)$;

T_old $(i)=(1-\bar{P}(i)) .{ }^{*}$ __star_old. ${ }^{*}(1-\mathrm{m} 1(i)) . / D$;

T_old_sum $=$ sum $\left(T \_o l d\right)$;

if T_old_sum>=1

end

break

end

TP_old_sum=sum(TP_old) $-T P \_o l d(i)$

for $j=1: 1: 100$

TP_new_l1 $(j)=-K-c \cdot{ }^{*} y \_s t a r \_n e w-$

d. ${ }^{*} y \_s t a r \_n e w+P(j) \cdot{ }^{*} \mathrm{~V} .{ }^{*} y \_s t a r \_n e w+(1-P(j)) \cdot{ }^{*} s .{ }^{*} y \_s t a r \_n e w . *(1-$

$m 1(j))+(1-P(j)) .{ }^{*} v \cdot{ }^{*} y \_s t a r \_n e w \cdot{ }^{*} m 1(j)$;

TP_new_12 $(j)=-(1-$

$P(j)) \cdot{ }^{*} y \_s t a r \_n e w .{ }^{*} \mathrm{cr} .{ }^{*} \mathrm{~m} 1(j)+P(j) \cdot{ }^{*} s \cdot{ }^{*} y \_s t a r \_n e w .{ }^{*} m 2(j)-$

$P(j) \cdot{ }^{*}$ y_star_new. ${ }^{*} \mathrm{ca} \cdot{ }^{*} \mathrm{~m} 2(j)$;

TP_new_13 $(j)=-1 . / 2 .{ }^{*}$ h. * $\left(\left(A^{\wedge} 2 . / D-\right.\right.$

D. $\left./\left(x^{\wedge} 2\right)+2 . / x\right) .{ }^{*}\left(y \_s t a r \_n e w \wedge 2\right)+(((1-$

$\left.\left.\left.P(j)) \cdot{ }^{*} P(j) .{ }^{*}\left(y \_s t a r \_n e w^{\wedge} 2\right) \cdot{ }^{*}(1-m 1(j)) \cdot{ }^{*} m 2(j)\right) \cdot / D\right)\right)$;

TP_new $(j)=T P \_n e w \_l 1(j)+T P \_n e w \_12(j)+T P \_n e w \_13(j)$;

T_new $(j)=(1-P(j)) \cdot{ }^{*}$ y_star_new. ${ }^{*}(1-m 1(j)) . / D$;

T_new_sum=sum(T_new);

if T_new_sum $>=1$

break

end

end

TP_new_sum=sum $\left(T P \_n e w\right)-T P \_n e w(j)$ 


\section{Appendix 4 Matlab code for the numerical comparison of Khan et al. (2014) and revised model}

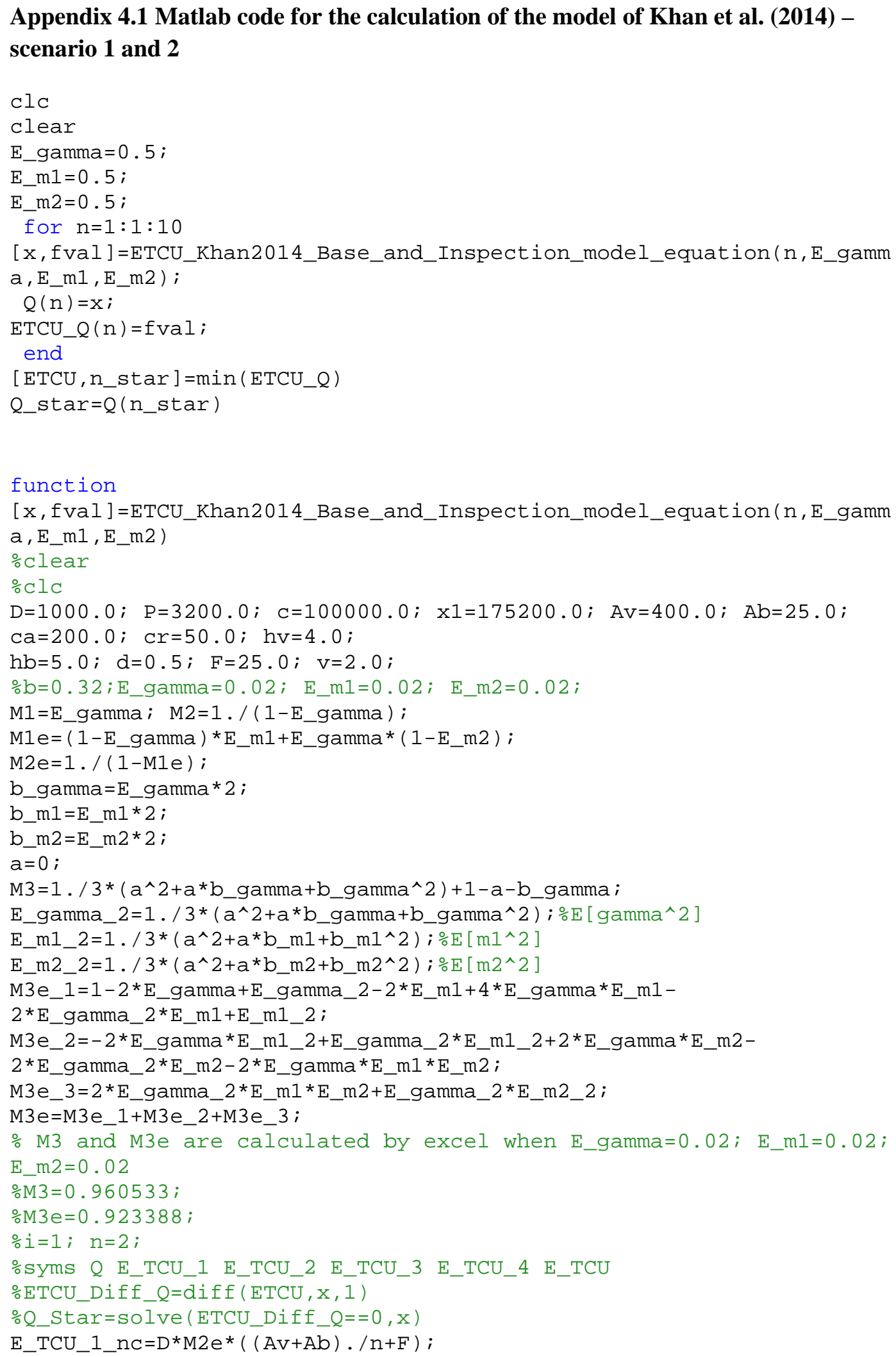




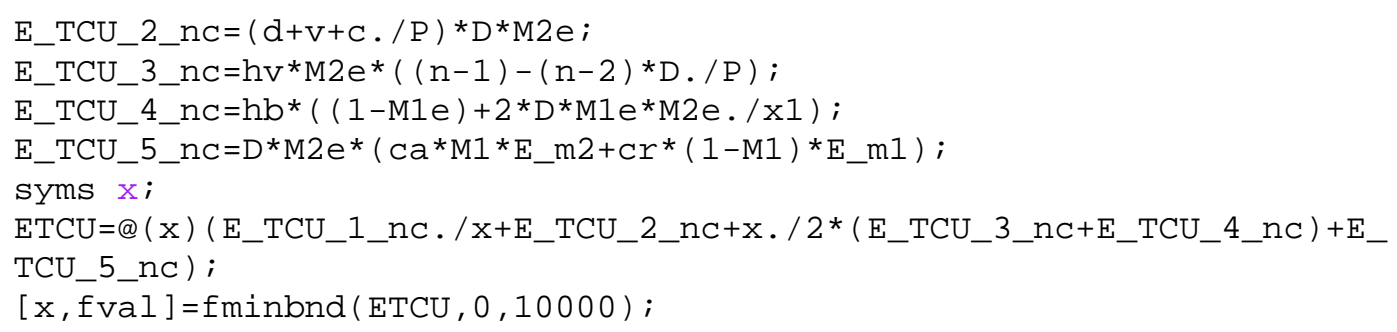

Appendix 4.2 Matlab code for the calculation of the model of Khan et al. (2014) scenario 3 and 4

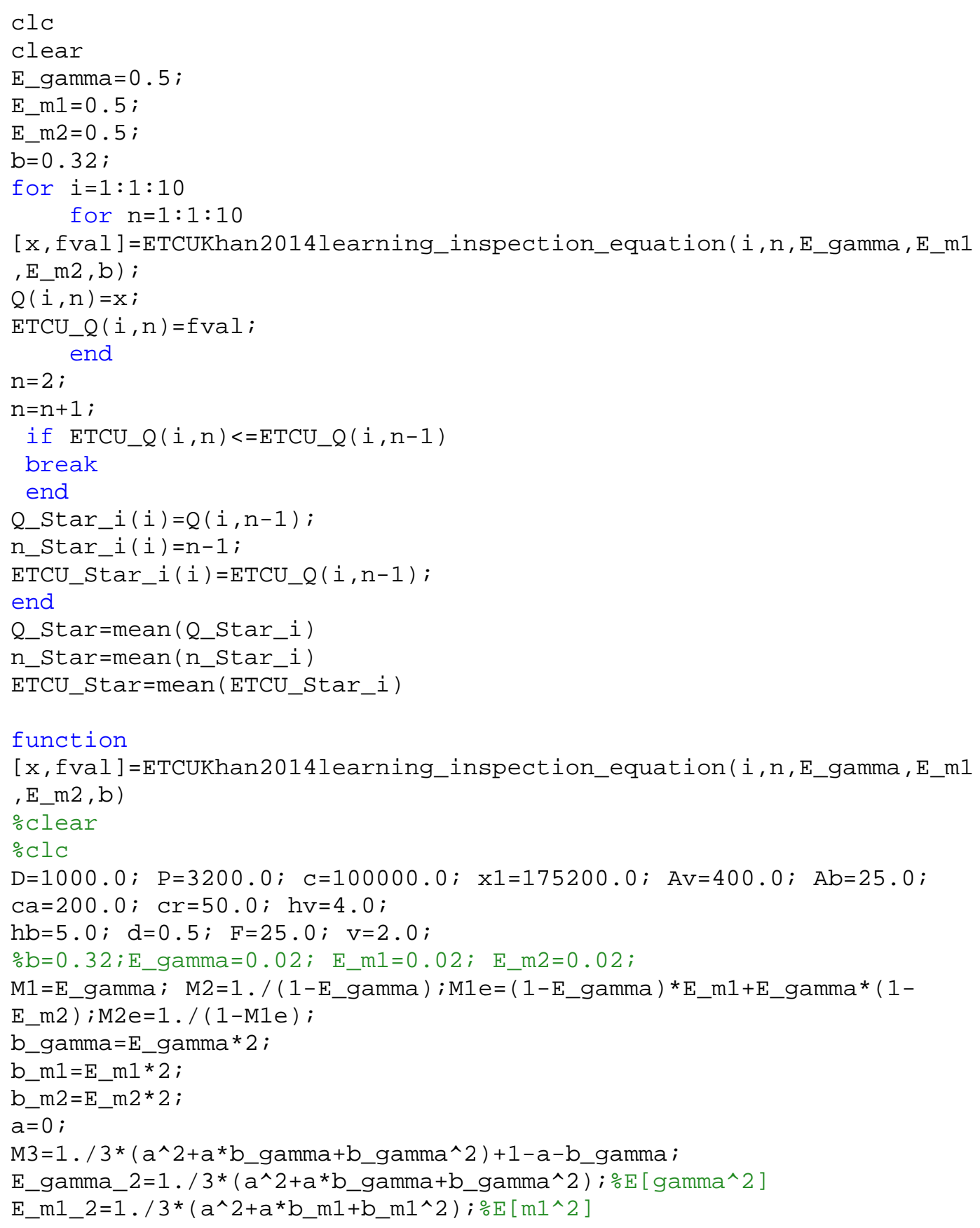




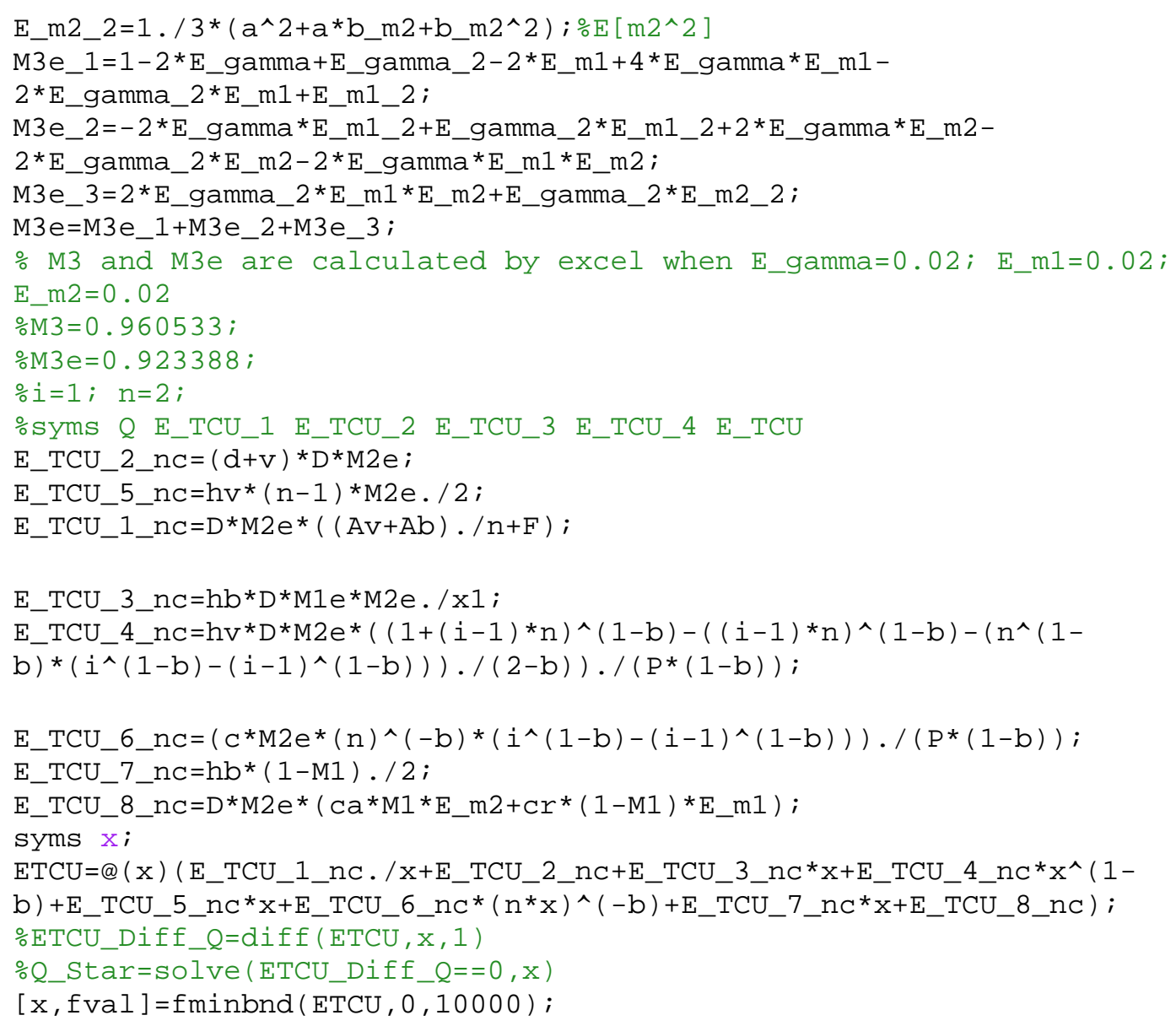

\section{Appendix 4.3 Matlab code for the calculation of the revised model of Khan et al.}

(2014) -scenario 1 and 2

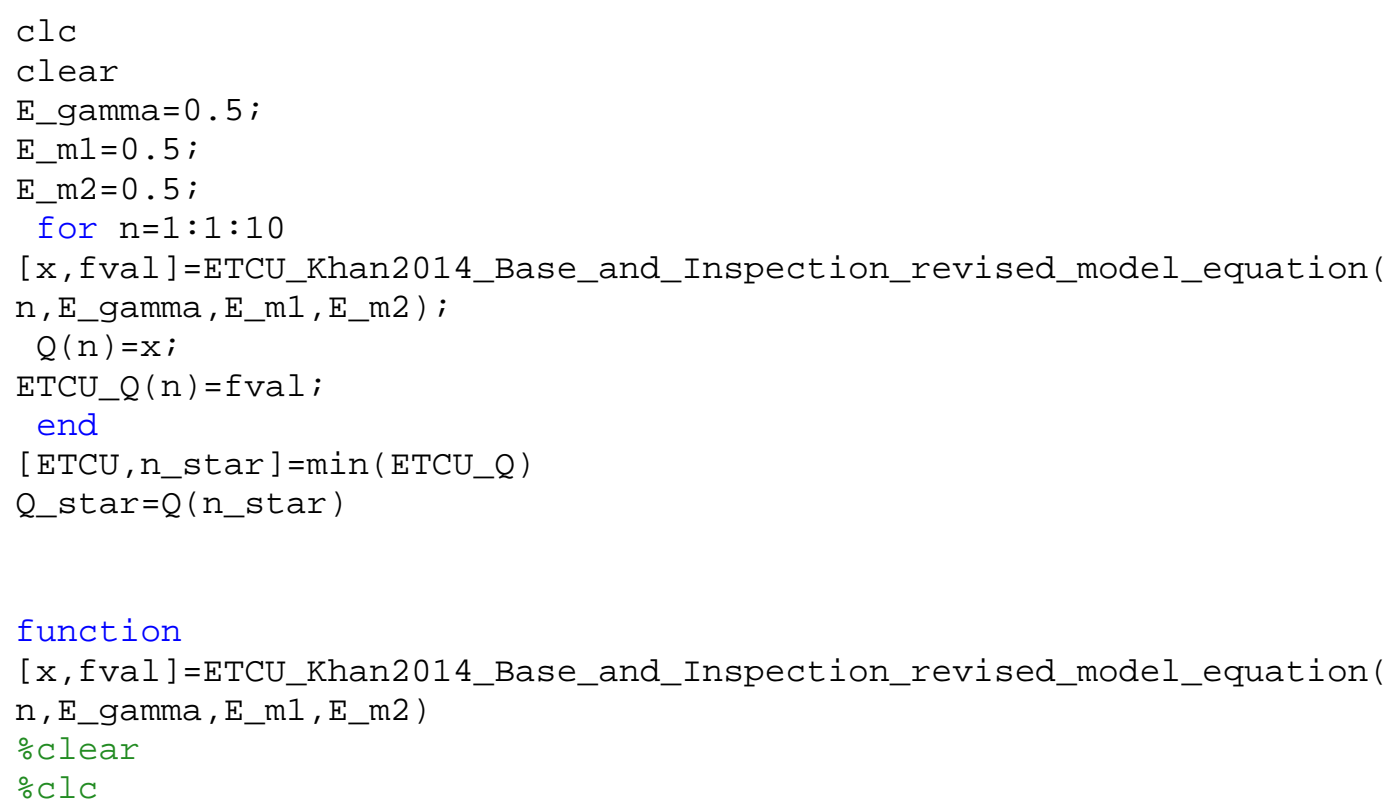




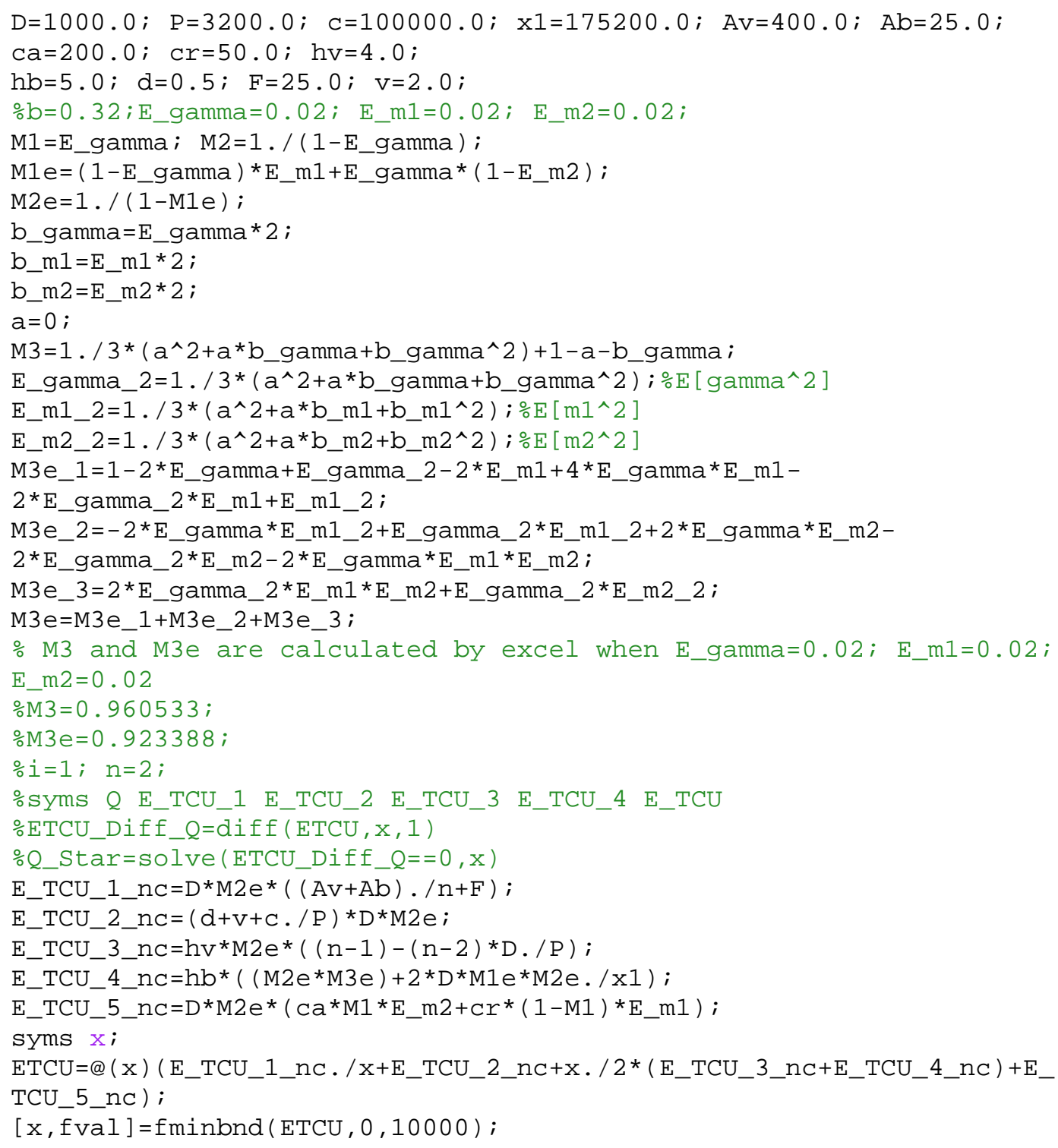

Appendix 4.4 Matlab code for the calculation of the revised model of Khan et al. (2014) -scenario 3 and 4

clc

clear

E_gamma $=0.5$;

E_m1 $=0.5$;

E_m2 $=0.5$;

$\mathrm{b}=0.32$;

for $i=1: 1: 10$

for $n=1: 1: 10$

[x, fval]=ETCUKhan2014learning_inspection_revised_equation(i,n,E_ga mma, E_m1, E_m2, b) ;

$\mathrm{Q}(\mathrm{i}, \mathrm{n})=\mathrm{x}$;

ETCU_Q $(i, n)=f v a l$; end

$\mathrm{n}=2 ; \mathrm{n}=\mathrm{n}+1$; 


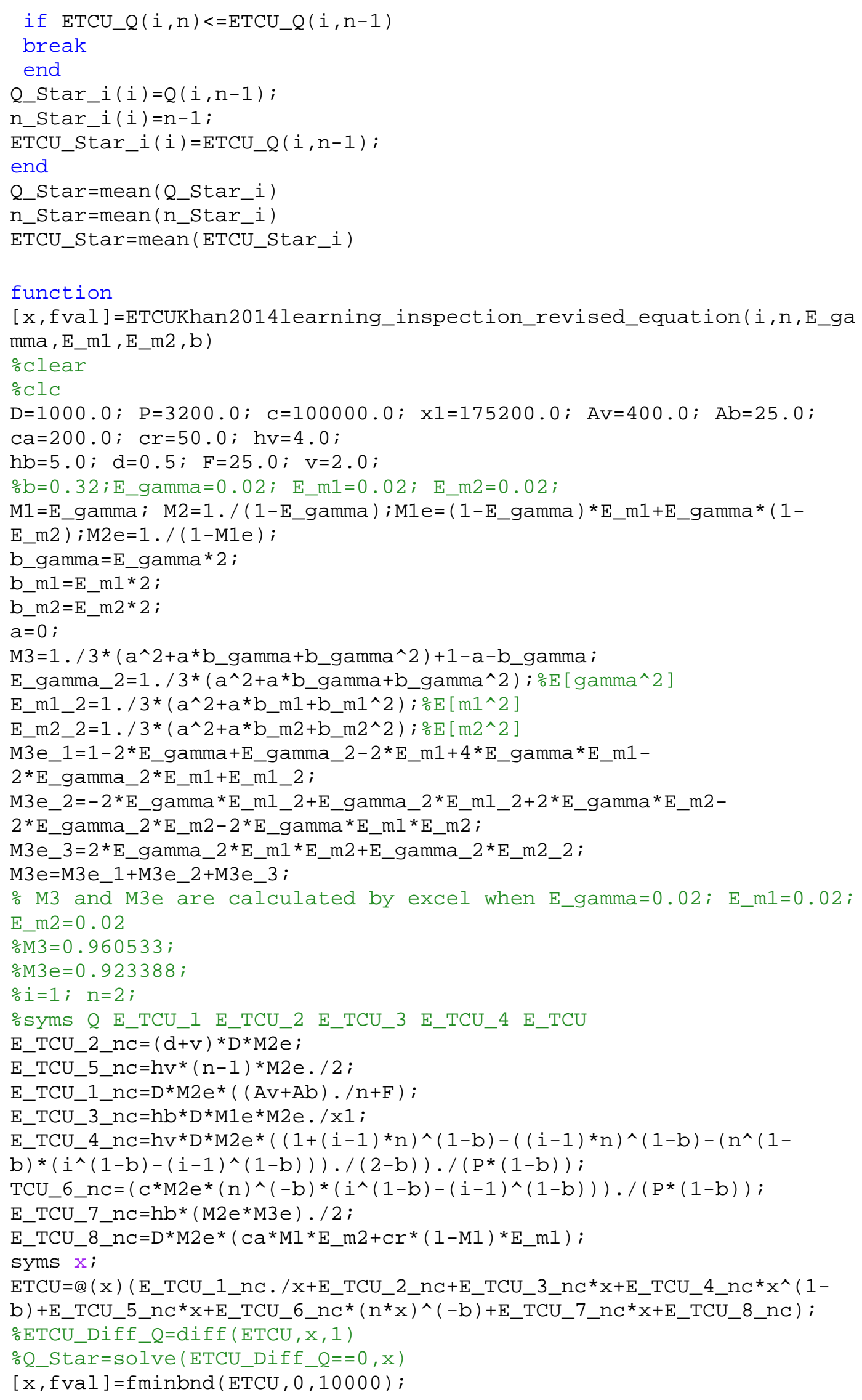




\section{References}

[1] Azadeh, A., Haghighi, S. M., Gaeini, Z. Shabanpour, N., 2016. Optimization of healthcare supply chain in context of macro-ergonomics factors by a unique mathematical programming approach. Applied Ergonomics, 55, 46-55.

[2] Chang, C.T., Cheng, M.C., Soong, P.Y., 2016. Impacts of inspection errors and trade credits on the economic order quantity model for items with imperfect quality. International Journal of Systems Science: Operations \& Logistics, 3(1), 34-48.

[3] Cardenas-Barron, L.E., 2000. Observation on: Economic production quantity model for items with imperfect quality. International Journal of Production Economics 67 (2), 201.

[4] Khan, M., Jaber, M.Y., Bonney, M., 2011. An economic order quantity (EOQ) for items with imperfect quality and inspection errors. International Journal of Production Economics 133, 113-118.

[5] Khan, M., Jaber, M.Y., Ahmad, A.R., 2014. An integrated supply chain model with errors in quality inspection and learning in production. Omega 42,16-24.

[6] Kuo-Lung Hou, K.L., Lin, L.C., Lin, T.Y., 2015. Optimal lot sizing with maintenance actions and imperfect production processes. International Journal of Systems Science, 46(15), 2749-2755.

[7] Maddah, B., Jaber, M.Y., 2008. Economic order quantity for items with imperfect quality: revisited. International Journal of Production Economics 112 (2), 808-815.

[8] Li, Q.D. ,2000. Application of soft experiment design in multi-factor sensibility analysis. Journal of Southwest Agricultural University 22(4),372-374 (in Chinese). 
[9] Rezaei, J., 2016. Economic order quantity and sampling inspection plans for imperfect items. Computers \& Industrial Engineering, 96, 1-7.

[10] Ross, A.M., 1996. Stochastic Processes, second ed. Wiley, New York, NY.

[11] Ross, A.M., 2009. Introduction to probability models, tenth ed. Academic Press in an Imprint of Elsevier.

[12] Salameh, M.K., Jaber, M.Y., 2000. Economic production quantity model for items with imperfect quality. International Journal of Production Economics 18 (6), 677-686.

[13] Teng, H.M., Hsu, P.M. Wee, H.M., 2013. Comments on “An economic order quantity (EOQ) for items with imperfect quality and inspection errors”. International Journal of Industrial Engineering Computations 4,173-176.

[14] Vörös, J., 2013. Economic order and production quantity models without constraint on the percentage of defective items. CEJOR 21, 867-885.

[15] Wolff, R. W., 1989. Stochastic Modeling and the Theory of Queues. Prentice _Hall, Englewood Cliffs, NJ.

[16] Zhou, Y.W., Chen, C.Y., Li, C.W., Zhong, Y.G, 2016. A synergic economic order quantity model with trade credit, shortages, imperfect quality and inspection errors. Applied Mathematical Modelling, 40(2), 1012-1028. 